\title{
Design and implementation of finite resolution crisp and fuzzy spatial objects
}

\author{
Markus Schneider \\ Department of Computer and Information Science and Engineering, University of Florida, Gainesville, \\ FL 32611-6120, USA
}

Received 4 April 2001; received in revised form 26 September 2001; accepted 4 June 2002

\begin{abstract}
Uncertainty management for geometric data is currently an important problem in spatial databases, image databases, and geographic information systems. Spatial entities do not always have homogeneous interiors and sharply defined boundaries but frequently their interiors and boundaries are partially or totally indeterminate and vague. For an important kind of spatial vagueness called spatial fuzziness this paper provides a conceptual and an implementation model of fuzzy spatial objects that also incorporates fuzzy geometric union, intersection, and difference operations as well as fuzzy topological predicates. In particular, this model is not based on Euclidean space and not on an infinite-precision arithmetic which lead to lacking numerical robustness and to topological inconsistency of implementations on a computer; it rests on a finite, discrete geometric domain called grid partition which takes into account finite-precision number systems available in computers. Last but not least, this paper is a contribution to achieve a uniform treatment of vector and raster data.
\end{abstract}

(C) 2002 Elsevier Science B.V. All rights reserved.

Keywords: Spatial database; Spatial fuzziness; Grid partition; Fuzzy spatial data type; Topological predicate

\section{Introduction}

Since the beginnings of a computational treatment of spatial data, the vector-raster debate has given rise to two different modeling directions: spatial data modeling views sets of objects in space and focuses on vector-oriented geometric data while image data modeling considers images of a

E-mail address: mschneid@cise.ufl.edu (M. Schneider). 
space and concentrates on digital, raster-oriented data. Modern geographical information systems (GIS) deal with both kinds of data but still have problems to present an integrated and uniform view of them.

Currently, spatial data modeling, as the design basis of spatial databases, is affected by two main problems. The first problem is that it has so far been suffering from the "boundary syndrome" which relates to the imagination that the extent of a spatial object is or has to be always limited by a precisely defined and abrupt boundary separating the interior of the object from its exterior. This view is certainly justified if we consider artifacts like land parcels with their cadastral boundaries, countries with their political boundaries, and districts with their administrative boundaries. But in general there is no apparent reason for the whole boundary of a region to be determined. There are many geographical application examples which illustrate that the boundaries of spatial objects like geological, soil, and vegetation units can be partially or totally indeterminate and blurred; many human concepts like "the Indian Ocean" or "South England" are implicitly vague.

The second problem is that spatial data modeling mainly rests on Euclidean space and Euclidean geometry and hence on an infinite-precision arithmetic. This conflicts with the reality of finite-precision number systems available in computers. For example, an intersection point of two lines has to be rounded to the nearest grid point where the grid corresponds to the resolution of the number system used. It is left to the implementor to close this gap between theory and practice. This leads inevitably not only to numerical but especially to topological errors and thus to wrong query results. Hence, it is recommendable to incorporate the aspect of finite representations explicitly into a spatial data model and to design finite resolution spatial data types that can be integrated as attribute types into databases.

Image data modeling as the basis of image databases and image processing suffers from the problem that previous attempts to design a consistent topological model for digital images have led to topological anomalies or have implied new unfavorable properties.

The goals of this paper are threefold: first, we provide a conceptual model of crisp and fuzzy spatial objects, namely fuzzy points, fuzzy lines, and fuzzy regions, and we investigate their topological properties. Second, we base our spatial objects on a finite resolution grid in order to take into account the discrete representations available in computers. These representations are especially interesting for fuzzy spatial objects since each contained, representable point has to be associated with a membership value. Third, this paper shall also be a contribution to achieve a uniform treatment of vector and raster data.

Section 2 compares with related work. Section 3 gives a unified view of vector and raster data, and Section 4 introduces a formalization of crisp and fuzzy points, lines, and regions based on a finite resolution grid. Section 5 gives a definition of the fuzzy geometric operations union, intersection, and difference on these types, and Section 6 deals with topological predicates for discrete regions. Section 7 deals with implementation aspects, and Section 8 draws some conclusions.

\section{Related work}

In this section we discuss related work about the different facets of spatial vagueness (Section 2.1), about previous work on fuzzy spatial objects (Section 2.2), and about finite resolution spatial data (Section 2.3). 


\subsection{Spatial vagueness}

Mainly two kinds of spatial vagueness [4] can be identified: spatial uncertainty is traditionally equated with randomness and chance occurrence and relates either to a lack of knowledge about the position and shape of an object with an existing, real boundary or to the inability of measuring such an object precisely. Spatial fuzziness is an intrinsic feature of an object itself and describes the vagueness of an object which certainly has an extent but which inherently cannot or does not have a precisely definable boundary (e.g., between a mountain and a valley). At least four alternatives have been proposed as general design methods: (1) exact models (for example, [5,13,21]) which transfer type systems and concepts for spatial objects with sharp boundaries to objects with unclear boundaries, (2) models based on rough sets [26] which work with lower and upper approximations of spatial objects, (3) probabilistic models (for example, [3,25]) which predominantly model positional and measurement uncertainty, and (4) models based on fuzzy sets (for example, $[1,3,8,23])$ which predominantly model fuzziness. The vagueness represented by fuzziness, in which we are only interested in this paper, does not describe the uncertainty of expectation like in probabilistic models but the vagueness resulting from the imprecision of the meaning of a concept. Examples of fuzzy spatial objects include mountains, valleys, air-polluted areas, biotopes, oceans, temperature zones, water-polluted rivers, magnetic fields, storm intensity, and sun insolation.

In image data modeling and image processing, in principle there has never been a problem to handle and to visualize values being of the same kind but having different intensity levels. Color shading and gray values are examples of such visualization methods. Thus, fuzziness has played a certain role. This can be seen from fuzzy digital topology [20], the fuzzy version of digital topology [19], which has been applied to pixel structures.

\subsection{Fuzzy spatial objects}

In [23] we have introduced spatial data types for fuzzy points, fuzzy lines, and fuzzy regions based on the Euclidean space. This data model is rather different from the one presented in this paper. It is an abstract model, does not take into account finite representations only available in computers, and is hence not implementable. Moreover, it is purely vector oriented. In [23] we have also given a classification of fuzzy regions from an application point of view. Core-boundary fuzzy regions suffer from insufficient knowledge about the grade of indeterminacy of the vague parts of a region. They rest on a three-valued logic and differentiate only between the core, the boundary, and the exterior of a region which relate to those parts that definitely belong, perhaps belong, and definitely do not belong, respectively, to the region. An application example is a lake which has a minimal water level in dry periods (core) and a maximal water level in rainy periods (boundary given as the difference between maximal and minimal water level). Finite-valued fuzzy regions lift the restriction of having only one degree of fuzziness and use a finite-valued (multi-valued) logic. This enables one to describe more precisely the degree (kind) of membership of a point in a fuzzy region. An application example are regions of different possibilities for virus infections. Regions could be categorized by $n$ different risk levels extending from areas with extreme risk of infection over areas with average risk of infection to safe areas. The two classes of fuzzy regions described so far have predominantly a qualitative character; the membership values only play a symbolic role here and are of lower importance. 
The next two classes have a quantitative character. Interval-based fuzzy regions partition the interval $[0,1]$ of possible membership values into a finite number of subintervals. This means that the degree of membership of each point lies somewhere between the borders of a subinterval, because we do not have more information. Smooth fuzzy regions take advantage of available knowledge about the distribution of attribute values within a fuzzy region. This knowledge can be gained by an expert through appropriate membership functions. We require that the distribution of attribute values within a fuzzy region is smooth (with a finite number of exceptions). In our discrete case we can, of course, only model gradual and not really (mathematically) continuous transitions within regions. We call this kind of fuzzy regions predominantly smooth (fuzzy) regions. As a special case we obtain (totally) smooth (fuzzy) regions with no "continuity gaps".

There are only few proposals of discrete fuzzy spatial data types. In [1] fuzzy regions are defined as fuzzy sets over $\mathbb{N}^{2}$. Each coordinate $(x, y) \in \mathbb{N}^{2}$ is associated with a value between 0 and 1 and describes the concentration of some feature attribute at that point. Unfortunately, the simple set property is insufficient since topological anomalies can arise, as we will see later. In a previous paper [24] we have already introduced some of our concepts for discrete fuzzy regions. These concepts have been partially changed and improved in this paper. In this sense, this paper is an improvement, extension, generalization, and completion of the previous one.

\subsection{Finite resolution spatial data}

Finite resolution spatial data, that is, spatial data defined over a discrete underlying geometric domain (for example, a homogeneous grid), has so far had a completely different significance for spatial and image information. It lies in the nature of image data modeling to handle discrete spatial data which is available as digital raster images. Digital topology (fuzzy digital topology) has been applied to describe the structure and the topological features of binary (finite-valued) images. In Section 3 we will show that these theories suffer from topological anomalies which make them inappropriate for our purposes. Digital topology has been used in [11] to model socalled 4-connected regions (see Section 3.2) with restricted conditions concerning their shape and size. From a modeling point of view, the shapes of these regions are very limited since their boundaries are two-dimensional. In particular the fact of two-dimensional boundaries leads to a number of topological configurations that would be inconsistent in $\mathbb{R}^{2}$.

In spatial data modeling hardly any work has so far been based on finite resolution data. Spatial data types (see [22] for an overview) proposed so far have all been based on exact twodimensional Euclidean space and have thus led to the problems of lacking numerical robustness and topological inconsistency. An exception is the realm concept [17,22]. A realm replaces the Euclidean space with a discrete geometric basis and is intended to represent the entire underlying geometry of an application. It is based on a finite resolution computational geometry [16] and consists of a finite set of points and line segments which are defined over a discrete grid and which form a spatially embedded planar graph. On top of realms a comprehensive and coherent spatial type system called the ROSE algebra [18,22] and a concept of vague regions [13] have been built. Each spatial object is described by a finite boundary representation and consists of a finite set of representable grid points. The ROSE algebra provides a very general definition of spatial data types for points, lines, and regions but does not include the modeling of image data. It has the nice property that it is closed under (appropriately defined) geometric union, intersection, and dif- 
ference operations. It allows lines to have a complex structure and to consist of several components; crisp regions may contain holes and islands within holes to any finite level. We would like to have these structure and closure properties for our discrete crisp and fuzzy spatial objects, too.

The expositions so far indicate that in the end both spatial and image data modeling have (especially conceptually) to deal with finite representations in order to guarantee implementability of spatial type systems in general and numerical robustness and topological consistency in particular. This understanding is a prerequisite for a uniform spatial information theory.

\section{Unifying the view on vector and raster data}

In most vector-oriented data models spatial objects are based implicitly on Euclidean space and geometry. In Section 3.1 we briefly summarize the insurmountable problems of this approach for implementation, and we discuss the transfer to a finite resolution and finite point set approach. At first glance it seems that this leads to a simple, common basis of vector and raster data. But the investigation of topological properties of parts of this common finite resolution basis reveals fundamental deficiencies of the underlying model. This, in particular, holds if we compare to the topological properties of spatial objects defined on the Euclidean space $\mathbb{R}^{2}$. Especially digital topology, which relates to the study of the topological properties of binary images, turns out to be not powerful enough and requires conceptual changes and extensions. This all is discussed in Section 3.2. Section 3.3 offers a solution to these problems which leads to the concept of grid partition. This concept rests on cell complexes as the basic construct of algebraic topology.

\subsection{Transforming vector data to finite resolution data}

Euclidean space and Euclidean geometry are very often used or implicitly assumed as the basis of spatial data models for vector data. But since they rest on an infinite-precision arithmetic (real numbers), they conflict with the reality of finite-precision number systems (integers, floating-point numbers) available in computers. We have already stressed that it is hence recommendable and essential to incorporate the aspect of finite representations explicitly into the spatial data model. The idea pursued in the following is to model spatial objects as finite point sets. Consequently, vector data has to be transformed into finite resolution data.

The first step of this transformation process is the same both for points, lines, and regions: we determine as the underlying discrete geometric domain a homogeneous grid given as a finite subset $\Omega=\{-n, \ldots, n\}^{2} \subset \mathbb{Z}^{2}$ with an arbitrary but fixed and representable $n \in \mathbb{N}$. An element of $\Omega$ is called a grid point having thus integer coordinates. This describes the grid view. For points this transformation to finite resolution data is trivial: a point is reduced to a grid point.

Lines and regions need two further transformation steps. In a second step, both kinds of objects are defined by boundary representations over this finite resolution grid so that their vertices are grid points and their segments are represented by pairs of grid points. This strategy has already been performed in the realm approach [17,22].

To get a better approximation of the boundary, in a third step, the Bresenham algorithm [2] is applied to each boundary segment. This algorithm describes a well-known method in computer graphics for computing a sequence of grid points to best represent a line segment whose end 


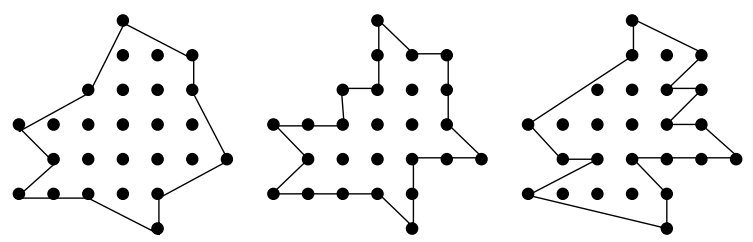

Fig. 1. An example of three different regions with the same finite point set.

points lie on grid points. A line can now be represented as the finite collection of points yielded by the vertices of the boundary representation and of the Bresenham algorithm. For regions the application of the Bresenham algorithm is possible but optional. A region is represented as the finite collection of points lying on and enclosed by the region's boundary representation. The mapping from boundary representations to finite point sets is usually not invertible, that is, given only the finite point set of a line or region, we are not able to uniquely derive its original boundary. As an example, Fig. 1 shows three different boundary representations of regions where the Bresenham algorithm has not been applied and which all enclose the same point set. If we assume a very high resolution (that is, a very large $n$ ) of $\Omega$, the perturbations resulting from this step become neglectible. We can then accept these slight perturbations and consider all lines and regions, respectively, with the same finite point set as being equal. Considering regions for instance, let REG be the set of all "well-defined" discrete (crisp) regions defined over $\Omega$, and let points $(u)$ be the mapping that yields the finite point set of $u \in \mathrm{REG}$. Then we can define an indiscernibility relation $\sim$ (which is an equivalence relation) on $\mathrm{REG}$ as follows: $\forall u, v \in \mathrm{REG}$ : $u \sim v \Longleftrightarrow \operatorname{points}(u)=$ points $(v)$. Somehow this is the necessary but acceptable price of finiteness that has to be paid for a uniform treatment of vector and raster data. If we intend to associate a boundary representation with a given finite point set of a region, we simply can take an arbitrary representative of the pertaining equivalence class. If two regions (of different equivalence classes) are adjacent, then corresponding neighbored boundary representations have to be selected.

It is certainly not surprising (because it has been deliberately modeled in this way) that the grid view corresponds to the pixel or raster view of digital images. In image data modeling, the requirement for finite representations is no problem since the concept of raster data fits in a natural way with discrete representations.

\subsection{Point set topology and the deficiencies of digital topology}

The ability to express topological properties of and between spatial objects (like connectivity, boundary, adjacency, etc.) is essential for the construction of spatial database systems, spatial query languages, and GIS. In the Euclidean space, point set topology [15] has turned out to be an appropriate theory to characterize topological relationships between spatial objects. Therefore, we would like to transfer its well known and desirable properties to an appropriate finite topology. A candidate for describing topological properties of finite point sets is digital topology [19]. It has been applied to raster images but lacks some important topological features which lead to "topological paradoxes". Below we give an overview of its most important concepts and demonstrate its main deficiencies. 
The starting point of point set topology is the notion of a topological space. Let $X$ be a set and $\mathscr{T} \subseteq 2^{X}$ be a subset of the power set of $X$. The pair $(X, \mathscr{T})$ is called a topological space if the axioms (i) $X \in \mathscr{T}, \emptyset \in \mathscr{T}$, (ii) $U \in \mathscr{T}, V \in \mathscr{T} \Rightarrow U \cap V \in \mathscr{T}$, and (iii) $U \subseteq \mathscr{T} \Rightarrow \bigcup_{A \in U} A \in \mathscr{T}$ are satisfied. $\mathscr{T}$ is called a topology for $X$. The elements of $\mathscr{T}$ are called open sets, their complements in $X$ closed sets. Point set topology mainly considers infinite point sets having the property that in an arbitrarily small neighborhood of a point infinitely many other points exist. This contradicts the nature of a discrete grid-based point whose neighborhood contains at most a finite number of other points. Two points in an infinite point set are connected if there exists a curve between them lying completely within the point set. Additionally, point set topology distinguishes different parts of a point set, namely its boundary $\partial A$, its interior $A^{\circ}$, and its exterior $A^{-}$, which are pairwise disjoint. The union of $A^{\circ}$ and $\partial A$ corresponds to the closure $\bar{A}$ of $A$. In $\mathbb{R}^{2}$ the Jordan curve theorem [15] is valid which states that a non-self-intersecting, closed, and continuous curve divides the Euclidean plane into two connected components, the interior and the exterior. If a point is removed from the curve, then the remainder of the plane becomes connected.

Digital topology is the study of the topological properties of discrete images and is therefore a possible candidate for modeling topological properties of crisp and fuzzy discrete spatial objects. But the following short summary reveals its fundamental deficiencies on a discrete domain compared to point set topology on a continuous domain. The underlying space of digital topology is the digital plane $\mathbb{Z}^{2}$. Let $S \subset \mathbb{Z}^{2}$. The points in $S$ are then called black points, and the points in $\mathbb{Z}^{2}-S$ are termed white points. Two main kinds of neighborhoods can be distinguished between grid points. The 4-neighbors of a point $(x, y)$ in $\mathbb{Z}^{2}$ are its four horizontal and vertical neighbors $(x+1, y),(x-1, y),(x, y+1)$ and $(x, y-1)$. The 8-neighbors of a point $(x, y)$ in $\mathbb{Z}^{2}$ are its four horizontal and vertical neighbors together with its four diagonal neighbors $(x+1, y+1)$, $(x+1, y-1),(x-1, y+1)$ and $(x-1, y-1)$. A 4-path (8-path) between any two points $p, q \in S$ is a sequence of points $p=p_{1}, \ldots, p_{n}=q$ with $p_{i} \in S$ such that $p_{i}$ is a 4-neighbor (8-neighbor) of $p_{i-1}, 1<i \leqslant n$. A path is called simple if each of its points is exactly once in the path. A set $S$ is 4 connected (8-connected) if for every pair of points $p, q$ of $S$ there is a 4-path (8-path) in $S$ from $p$ to q. A 4-component (8-component) of a set $S$ is a greatest 4-connected (8-connected) subset of $S$.

Attempts to develop a consistent topology of two-dimensional discrete images by means of these two notions of neighborhood have failed due to so-called "topological paradoxes". We here illustrate the connectivity paradox [19] and show that the Jordan Curve Theorem is neither valid for 4-neighborhood nor for 8-neighborhood. A digital version of the Jordan Curve Theorem would imply to replace a simple closed curve of the Euclidean plane by a simple closed path of the digital plane. Unfortunately, the set of grid points not belonging to the closed path is not always separated into two components. If in Fig. 2a 8-neighborhood is used for all pairs of points, then the black points form a discrete analog of a Jordan curve (simple closed path) but they do not separate the white points. The situation is not better for 4-neighborhood. The black points in Fig. $2 \mathrm{~b}$ determine a 4-connected simple closed path but there exist three 4-connected components for the remaining white points. Thus a digital version of the Jordan Curve Theorem holds in neither case. Moreover, if in Fig. 2a 4-neighborhood is used for all pairs of points, then the black points are completely disconnected but still separate the set of white points into two components. If 4neighborhood is used for all pairs of points and we have a 4-connected simple closed path like in Fig. 2c, then the white points remain separated if we remove a corner (circled point) from the path. 


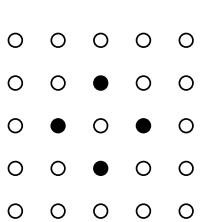

(a)

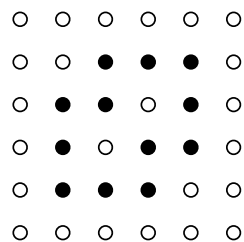

(b)

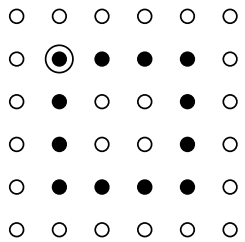

(c)

Fig. 2. Examples of connectivity paradoxes.

The main problem of digital topology is that it is "hand-made" and that it does not refer to the definition of a topological space. Hence, it is impossible to transfer important topological notions like open set, open neighborhood, continuity, and many others, to a two-dimensional grid-based domain. Another drawback is that boundaries are two-dimensional structures, a fact that does not correspond to our expectation of spatial reality.

\subsection{The unification step: grid partitions}

The deficiencies of digital topology can be remedied if we return to classical topology. Then a solution comprises two main aspects: first, we form unit areas which relate to the regions between four adjacent grid points and which contain all and only their interior and boundary points. This measure ensures the existence of an underlying topological space and the avoidance of geometric anomalies. The consequence is that the intersection of two unit areas is either empty (if they are disjoint), or a zero-dimensional point (if they meet at a corner), or a one-dimensional unit segment (if they share a unit segment), or a two-dimensional unit area (if they are identical). Hence, second, we have to consider the digital plane as a structure consisting of elements of different dimensions. Such a structure is well known in algebraic topology as a cellular complex [6] and has already been used in spatial data modeling (for example, $[10,14]$ ) for decomposing space into a collection of irregular geometric shapes. We first briefly discuss some of the most important definitions of algebraic topology, show why the connectivity paradox cannot arise, and then introduce the notion of grid partition.

\subsubsection{Cells and cellular complexes}

A homeomorphism [6] between two spatial objects is an invertible function from one object to another such that both the function and its inverse are continuous. This essentially means that there is a mapping of the points in the first object to the points in the second one (and vice versa) that preserves the concept of proximity. The closed $n$-disc with center $x$ and radius $\varepsilon \in \mathbb{R}^{+}$is the set of points in the $n$-dimensional Euclidean space with a distance from $x$ less than or equal to $\varepsilon$. A closed $n$-cell is any spatial object homeomorphic (that is, topologically equivalent) to the closed $n$ disc. The surface of the closed $(n+1)$-disc with radius $\varepsilon$ describes all points at a distance of exactly $\varepsilon$ and is called the $n$-sphere. The boundary of an $n$-cell is that part of the $n$-cell mapped onto by the $(n-1)$-sphere by any homeomorphism. The interior of an $n$-cell is that part of its $n-1$-cells that do not belong to the boundary. An open $n$-cell is a closed $n$-cell without boundary. Examples of 0 cells are points, examples of 1-cells are continuous curves and straight segments, and examples of 


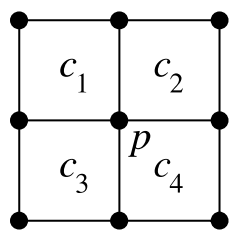

Fig. 3. Considering 0 -cells contributes to solving the connectivity paradox.

2-cells are circles, triangles, and polygons. The boundary of a triangle, for example, consists of its three bounding straight segments. The boundary of each segment contains its two end points. All $k$-cells of an $n$-cell for $0 \leqslant k<n$ are called faces of the $n$-cell. A $k$-cell is said to bound an $n$-cell with $0 \leqslant k<n$ if the $k$-cell is a face of the $n$-cell.

An $n$-dimensional cellular complex ( $n$-complex, $n$-cell complex) is a collection $C$ of $n$-cells such that (i) $C$ contains all faces of all elements of $C$ (called completeness of inclusion in [14]), and (ii) the intersection of two cells in $C$ is either empty or a face of both cells (called completeness of incidence in [14]). These two conditions correspond to the definition of a (finite) topological space so that a cellular complex is a finite topological space. From a data type point of view, this means that cellular complexes are closed under the set operations union, intersection, difference, and complement. ${ }^{1}$ An $n$-complex of special interest is the punctured cell consisting of an $n$-cell from which a smaller $n$-cell has been cut out. In two-dimensional space this is homeomorphic to an annulus.

We can also express the topological property of connectivity for cellular complexes. A sequence of elements of a subset $S$ of a cellular complex $C$ beginning at $c_{1}$ and finishing at $c_{2}$ is called a path in $S$ from $c_{1}$ to $c_{2}$ if for every two neighbored elements in the sequence one of them bounds the other. $S$ is called connected if for any two elements $c_{1}, c_{2} \in S$ a path in $S$ from $c_{1}$ to $c_{2}$ exists. We can now argue why a connectivity paradox cannot occur here. Let $C$ be a cellular complex containing the 2-cells $c_{1}, c_{2}, c_{3}$ and $c_{4}$ (Fig. 3). A subcomplex $S$ including $c_{1}$ and $c_{4}$ but not $c_{2}$ and $c_{3}$ can only be connected if and only if the 0 -cell $p \in S$, since a path in $S$ from $c_{1}$ to $c_{4}$ has to pass $p$. But if $p \in S$, then $p \notin C \backslash S$, and therefore $C \backslash S$ is not connected. Assigning the 0-cell $p$ either to $S$ or to its complement avoids the connectivity paradox since otherwise $S$ and $C \backslash S$ could be both connected or both disconnected. Hence, the connectivity paradox can only arise if 0 -cells and 1cells are ignored.

\subsubsection{Grid partitions}

Actually, we return to the vector model of spatial databases where we can distinguish one-, zero-, and two-dimensional spatial elements, too. In our case we use cells and cell complexes to obtain a regular cellular decomposition of the plane which we call grid partition. The grid partition restricts the set of possible structures in $\mathbb{R}^{2}$ to collections of regularly shaped grid units. This measure transfers the topological properties of $\mathbb{R}^{2}$ to the grid partition, because the grid partition

\footnotetext{
${ }^{1}$ An interesting observation is that a realm [17,22] also forms a cellular complex and that it hence is a finite topological space.
} 
is embedded into the continuous Euclidean plane being a topological space. Hence, the grid partition turns out to be able to replace the image (or raster) model of $\mathbb{Z}^{2}$.

We now define the notion of a grid unit as the central component of a grid partition. Let $q=(x, y) \in \mathbb{R}^{2}$, and let $v$ be a normalization function with $v(x, y)=\left(x+\frac{1}{2}, y+\frac{1}{2}\right)$. We extend $v$ to subsets $A \subseteq \mathbb{R}^{2}$ by $v(A)=\{v(q) \mid q \in A\}$. For $q, r \in \mathbb{R}^{2}$ let $] q, r[$ denote the open straight segment between $q$ and $r$. Moreover, for a point $p=(i, j) \in \Omega$ with $i, j \notin\{-n, n\}$ we define $p_{0}(i, j)=$ $\left(i-\frac{1}{2}, j-\frac{1}{2}\right), p_{1}(i, j)=\left(i+\frac{1}{2}, j-\frac{1}{2}\right), p_{2}(i, j)=\left(i+\frac{1}{2}, j+\frac{1}{2}\right)$, and $p_{3}(i, j)=\left(i-\frac{1}{2}, j+\frac{1}{2}\right)$. The grid unit for a point $p=(i, j) \in \Omega$ is the triple $G(p)=(C(p), E(p), V(p))$ such that

(i) $C(p)=\left\{v(] i-\frac{1}{2}, i+\frac{1}{2}[\times] j-\frac{1}{2}, j+\frac{1}{2}[)\right\}$

(ii) $E(p)=\left\{v(] p_{t}(i, j), p_{(t+1) \bmod 4}(i, j)[) \mid 0 \leqslant t \leqslant 3\right\}$

(iii) $V(p)=\left\{v\left(p_{t}(i, j)\right) \mid 0 \leqslant t \leqslant 3\right\}$

Note that $C(p), E(p)$, and $V(p)$ are finite sets with $|C(p)|=1$ and $|E(p)|=|V(p)|=4$. Since the points $p_{t}(i, j)$ are not elements of $\Omega$ and are thus not representable in our discrete domain, the function $v$ performs a homeomorphic translation operation to representable coordinates in $\Omega$. Hence, each point $(i, j)$ is mapped to the grid unit (unit square) $G(i, j)$ with the left lower bound $(i, j)$ and the right upper bound $(i+1, j+1)$. A grid unit consists of three parts: $C(p)$ contains its axis-parallel, open quadrangle of unit length 1 (called unit area) as a 2-cell and is modeled as a singleton set. To determine the infinite point set represented by this finite representation of a 2-cell we need a further notation. Let $A, B$ be sets and $f: A \rightarrow \mathscr{P}(B)$ be a function. If we are sure that, for example, $f(a)$ yields a singleton set, we write $f^{!}(a)$ to denote this single element, that is, $f(a)=$ $\{b\} \Rightarrow f^{!}(a)=b$. Now we can express the infinite point set represented by $C(p)$ as $p n t s(C(p)):=$ $C^{!}(p) . E(p)$ contains its four axis-parallel, open unit segments (called edges) as 1-cells, and the corresponding infinite point set is pnts $(E(p)):=\bigcup_{S \in E(p)} S . V(p)$ contains its four corners (called points or vertices) as 0-cells, and the corresponding (finite) point set is pnts $(V(p)):=V(p)$. We have deliberately defined that every $k$-cell is open and does hence not contain its boundary, that is, pnts $(C(p)) \cap p n t s(E(p)) \cap \operatorname{pnts}(V(p))=\emptyset$. Thus, each represented point of $G(p)$ belongs either to a 0 -cell, a 1-cell, or a 2-cell. The point set pnts $(C(p)) \cup \operatorname{pnts}(E(p)) \cup p n t s(V(p))$ describes the complete represented point set of the grid unit. Fig. 4a shows the structure of a grid unit as a cellular complex. The grid partition ${ }^{2}$ over $\Omega$ is the set $G(\Omega)=\{G(p) \mid p \in \Omega\}$. Moreover, we define $C(\Omega)=\bigcup_{p \in \Omega} C(p), E(\Omega)=\bigcup_{p \in \Omega} E(p)$, and $V(\Omega)=\bigcup_{p \in \Omega} V(p)$. An analogous definition holds for $G\left(\mathbb{Z}^{2}\right)$. Fig. $4 \mathrm{~b}$ shows an example of a grid partition as a cellular complex.

In summary one can say that in a two-stage process we have substituted the digital plane $\mathbb{Z}^{2}$ for the Euclidean plane $\mathbb{R}^{2}$ and the grid partition $G\left(\mathbb{Z}^{2}\right)$ due to topological weaknesses for the digital plane $\mathbb{Z}^{2}$. The result is a regular cellular decomposition of $\mathbb{R}^{2}$ (or a regular realm in the sense of $[18,22])$, which is also discrete like $\mathbb{Z}^{2}$, but moreover preserves the topological features of $\mathbb{R}^{2}$. In image modeling the 2-cells of $G\left(\mathbb{Z}^{2}\right)$ correspond to pixels. We have here the problem that 0 -cells and 1-cells are not realized in hardware devices like monitor screens and image memories, but grid

\footnotetext{
${ }^{2}$ The notion of grid partition differs from the notion of spatial partition (map, coverage) given, for example, in [12]. In a spatial partition, boundaries of adjacent areas with the same attribute are eliminated, and the areas are merged together.
} 


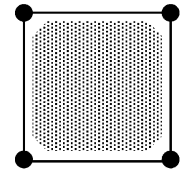

(a)

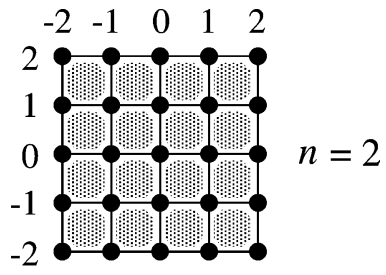

(b)

Fig. 4. A grid unit as a 2-cell (a) and a grid partition as a 2-cell complex (b).

partitions can at least serve as a conceptual model for topologically consistent pictures in image processing. In spatial (that is, vector) modeling, the conceptual extensions are realizable without difficulties.

\section{Finite resolution spatial objects}

In this section, in a very general way we define spatial data types for crisp and fuzzy point objects, line objects, and region objects as parts of the discrete geometric domain $G(\Omega)$. Generality here especially implies that the data types are closed under the geometric operations union, intersection, difference, and complement (as well as under other spatial operations). For example, a point object contains collections of points and not only single points. This is a desirable property for the maintenance of closure properties since, for instance, the intersection of two line objects usually just yields a collection of points (besides a collection of line segments). A region object may have holes and may consist of several components.

Section 4.1 introduces some basic concepts of fuzzy set theory, as far as they are relevant in this context, and discusses discrete membership functions. The next subsections deal with crisp and fuzzy points (Section 4.2), crisp and fuzzy lines (Section 4.3), and crisp and fuzzy regions (Section 4.4). It turns out that the crisp data types are always special instances of the pertaining fuzzy data types.

\subsection{Fuzzy sets and discrete membership functions}

Fuzzy set theory [27] is an extension and generalization of Boolean set theory. Let $X$ be a classical (crisp) set of objects, called the universe (of discourse). Membership in a classical subset $A$ of $X$ can then be described by the characteristic function $\chi_{A}: X \rightarrow\{0,1\}$ such that for all $x \in X$ holds $\chi_{A}(x)=1$ if and only if $x \in A$ and $\chi_{A}(x)=0$ otherwise. This function, which discriminates sharply between members and non-members of a set, can be generalized such that all elements of $X$ are mapped to the real interval $[0,1]$ indicating the degree of membership of these elements in the set in question. Hence, fuzzy set theory permits an element to have partial and multiple membership. Larger values designate higher grades of set membership. We call $\mu_{\tilde{A}}: X \rightarrow[0,1]$ the membership function of $\tilde{A}$, and the set $\tilde{A}=\left\{\left(x, \mu_{\tilde{A}}(x)\right) \mid x \in X\right\}$ is called a fuzzy set in $X$. All elements of $X$ receive an assessment with respect to their membership in $\tilde{A}$. Those elements $x \in X$ that in the classical sense do not belong to $\tilde{A}$ get the membership value $\mu_{\tilde{A}}(x)=0$; elements $x \in X$ that completely belong to $\tilde{A}$ get the membership value $\mu_{\tilde{A}}(x)=1$. We also allow the notations $\tilde{x}:=$ $\left(x, \mu_{\tilde{A}}(x)\right) \in \tilde{A}$ and $\mu(\tilde{x}):=\mu_{\tilde{A}}(x)$. 
There are many ways of extending the crisp set inclusion as well as the basic crisp set operations to fuzzy sets. We follow the definitions in [27]. Let $\tilde{A}$ and $\tilde{B}$ be fuzzy sets in $X$. Then

(i) $\neg \tilde{A}=\left\{\left(x, \mu_{\neg \tilde{A}}(x)\right) \mid x \in X, \mu_{\neg \tilde{A}}(x)=1-\mu_{\tilde{A}}(x)\right\}$

(ii) $\tilde{A} \subseteq \tilde{B} \Longleftrightarrow \forall x \in X: \mu_{\tilde{A}}(x) \leqslant \mu_{\tilde{B}}(x)$

(iii) $\tilde{A} \cap \tilde{B}=\left\{\left(x, \mu_{\tilde{A} \cap \tilde{B}}(x)\right) \mid x \in X \wedge \mu_{\tilde{A} \cap \tilde{B}}(x)=\min \left(\mu_{\tilde{A}}(x), \mu_{\tilde{B}}(x)\right)\right\}$

(iv) $\tilde{A} \cup \tilde{B}=\left\{\left(x, \mu_{\tilde{A} \cup \tilde{B}}(x)\right) \mid x \in X \wedge \mu_{\tilde{A} \cup \tilde{B}}(x)=\max \left(\mu_{\tilde{A}}(x), \mu_{\tilde{B}}(x)\right)\right\}$

(v) $\tilde{A}-\tilde{B}=\tilde{A} \cap \neg \tilde{B}$

A [strict $] \alpha$-cut or [strict $] \alpha$-level set of a fuzzy set $\tilde{A}$ for a specified value $\alpha$ is the crisp set $A_{\alpha}\left[A_{\alpha}^{*}\right]=\left\{x \in X \mid \mu_{\tilde{A}}(x) \geqslant[>] \alpha \wedge 0 \leqslant \alpha \leqslant[<] 1\right\}$. The strict $\alpha$-cut for $\alpha=0$ is called support of $\tilde{A}$, i.e., $\operatorname{supp}(\tilde{A})=A_{0}^{*}$. For a fuzzy set $\tilde{A}$ and $\alpha, \beta \in[0,1]$ we obtain $X=A_{0}$ and $\alpha<\beta \Rightarrow A_{\alpha} \supseteq A_{\beta}$. The set of all levels $\alpha \in[0,1]$ that represent distinct $\alpha$-cuts of a given fuzzy set $\tilde{A}$ is called the level set $\Lambda_{\tilde{A}}$ of $\tilde{A}: \Lambda_{\tilde{A}}=\left\{\alpha \in[0,1] \mid \exists x \in X: \mu_{\tilde{A}}(x)=\alpha\right\}$.

Since we are aiming at finite representations, the definition of a membership function is too general. The universe of discourse we are interested in is the finite grid partition $X=G(\Omega)$. Because the interval $[0,1]$ represents an infinite set of membership values, we have to restrict it to a finite and thus representable set $\Lambda=\left\{\alpha_{1}, \ldots, \alpha_{m}\right\}$ for some $m \in \mathbb{N}$ with $0=\alpha_{1}<\alpha_{2}<\cdots<$ $\alpha_{m-1}<\alpha_{m}=1$. For each fuzzy set $\tilde{A}$ in $G(\Omega)$ then $\Lambda_{\tilde{A}} \subseteq \Lambda$ holds, and $\Lambda=\bigcup\left\{\Lambda_{\tilde{A}} \mid \tilde{A}\right.$ is a fuzzy set in $G(\Omega)\}$. We also introduce a special notation for subsets of the power set $\mathscr{P}(\Lambda)$ containing a particular element $\alpha_{k} \in \Lambda$ and being of constrained size: for $1 \leqslant k \leqslant m$ and $t \in \mathbb{N}$ we define $\Lambda^{k, t}:=\left\{A \in \mathscr{P}(\Lambda)\left|\alpha_{k} \in A, 1 \leqslant\right| A \mid \leqslant t\right\}$.

Accordingly, in our context we have discrete membership functions, and the discrete membership function for any fuzzy set $\tilde{A}$ is here defined as $\mu_{\tilde{A}}:(C(\Omega) \cup E(\Omega) \cup V(\Omega)) \rightarrow \mathscr{P}(\Lambda)$. This means that a grid unit is always associated with a set of membership values, which we here also call labels. Except for the fact that the selection of labels for all fuzzy spatial objects is application dependent, it is arbitrary for the 0-cells of fuzzy points. We will see that this is not the case for the cells of fuzzy lines and fuzzy regions due to the topological interdependence of 0 -cells, 1-cells, and 2-cells of the units of the grid partition. We are confronted with the problem of appropriately assigning membership values to the vertices, edges, and unit areas of a fuzzy spatial object. This problem will be treated in Section 4.3 for fuzzy lines and in Section 4.4 for fuzzy regions.

Another important aspect motivated by spatial phenomena like air pollution or temperature distributions is that we are interested in modeling "smooth" or "continuous" transitions within the interiors of lines and regions. This feature resembles different grey values in images which are used to visualize different levels of intensity of an attribute.

\subsection{Crisp and fuzzy points}

A crisp point is an element of $V(\Omega)(=\Omega)$. A fuzzy point is also an element of $V(\Omega)$, but it is annotated with one of the $m-1$ membership values greater than 0 , since 0 documents the nonexistence of a point. A fuzzy point $\tilde{p}$ at $(a, b)$ in $V(\Omega)$, written $\tilde{p}(a, b)$, is a fuzzy singleton in $V(\Omega)$ defined by 


$$
\mu_{\tilde{p}(a, b)}(x, y)= \begin{cases}\left\{\alpha_{i}\right\} & \text { if }(x, y)=(a, b) \\ 0 & \text { otherwise }\end{cases}
$$

with $\left\{\alpha_{i}\right\} \subseteq \Lambda \backslash\left\{\alpha_{1}\right\}$. Point $\tilde{p}$ is said to have support $(a, b)$ and value $\left\{\alpha_{i}\right\}$. Let $V_{f}(\Omega)=$ $\left\{\tilde{p} \mid \operatorname{supp}(\tilde{p}) \in V(\Omega), \mu(\tilde{p}) \subseteq \Lambda \backslash\left\{\alpha_{1}\right\}\right\}$ be the set of all fuzzy points over $V(\Omega)$. Note that we have generalized the meaning of a fuzzy point in the sense that it may carry more than one label. This happens, for instance, if a fuzzy point has two or more incident edges with different labels in a fuzzy line (see Section 4.3). A single and isolated fuzzy point, however, always carries a single label. $V_{f}(\Omega)$ is, of course, finite and a proper superset of the set $V(\Omega)$ of all crisp points. If we are sure that, for example, $\mu_{\tilde{p}(a, b)}$ yields a singleton set, we write $\mu_{\tilde{p}(a, b)}^{!}$to denote this single element, that is, $\mu_{\tilde{p}(a, b)}=\{\alpha\} \Rightarrow \mu_{\tilde{p}(a, b)}^{!}=\alpha$. For $\tilde{p}=p=(a, b) \in V(\Omega)$, we then obtain $\mu_{\tilde{p}(a, b)}^{!}(x, y)=$ $\chi_{p}(x, y)=1$, if $(x, y)=(a, b)$, and 0 otherwise.

Next, we define four important comparison operators on fuzzy points. Let $\tilde{p}(a, b), \tilde{q}(c, d) \in$ $V_{f}(\Omega)$ with $(a, b),(c, d) \in V(\Omega)$. Then

(i) $\tilde{p}(a, b)=\tilde{q}(c, d): \Longleftrightarrow a=c \wedge b=d \wedge \mu_{\tilde{p}(a, b)}=\mu_{\tilde{q}(c, d)}$

(ii) $\tilde{p}(a, b) \neq \tilde{q}(c, d): \Longleftrightarrow \neg(\tilde{p}(a, b)=\tilde{q}(c, d))$

(iii) $\tilde{p}(a, b)<\tilde{q}(c, d): \Longleftrightarrow \operatorname{supp}(\tilde{p})<\operatorname{supp}(\tilde{q}) \vee\left(\operatorname{supp}(\tilde{p})=\operatorname{supp}(\tilde{q}) \wedge \max \left(\mu_{\tilde{p}(a, b)}\right)<\max \left(\mu_{\tilde{q}(c, d)}\right)\right)$

(iv) $\tilde{p}(a, b)<\mu_{\tilde{q}(c, d)}: \Longleftrightarrow \max \left(\mu_{\tilde{p}(a, b)}\right)<\max \left(\mu_{\tilde{q}(c, d)}\right) \vee\left(\max \left(\mu_{\tilde{p}(a, b)}\right)=\max \left(\mu_{\tilde{q}(c, d)}\right) \wedge \operatorname{supp}(\tilde{p})<\right.$ $\operatorname{supp}(\tilde{q}))$

We see that at least two different order relations can be defined on discrete points: the first one (iii) especially takes the lexicographical order of the points into account, and the second one (iv) emphasizes the importance of the points' membership values.

We are now able to define spatial data types for fuzzy and crisp points. The definition of the fuzzy spatial data type fpoint is as follows:

$$
\text { fpoint }=\mathscr{P}\left(V_{f}(\Omega)\right)
$$

where $\mathscr{P}(Y)$ denotes the power set of a set $Y$. Let $1_{f}=V(\Omega) \times\{1\}$. Then we can simply define the type point for crisp points as:

$$
\text { point }=\mathscr{P}\left(1_{f}\right)
$$

Of course, the cardinality of fpoint and point is finite, and obviously, point $\subset$ fpoint holds.

\subsection{Crisp and fuzzy lines}

In this section we are especially interested in a "structured" view of discrete crisp and fuzzy lines. Their definition is based on the observation that the collection $V(\Omega) \cup E(\Omega)$ represents a spatially embedded planar graph. This means that two edges of two line objects are either disjoint, or they coincide, or they are connected by a common vertex of $V(\Omega)$. In particular, they do not properly intersect each other since all intersection points between lines are known in advance. Or we could also say that all line objects definable over $G(\Omega)$ have already become acquainted with each other. This property contributes to a numerically robust and topologically consistent behavior of objects and operations. Consequently, line objects are constructed from the 0 -cells and 1-cells of the grid partition. 
Intuitively we regard a simple line as a sequence of edges without inner cycles. Formally it will be defined as an alternating connected sequence of points and edges, since each edge is bounded by two points. The only open issue in the definition relates to the global labeling strategy for points and edges of a simple fuzzy line. For example, consider two edges bounded by a common vertex, and let us assume that both edges are labeled differently. Which label should then be attached to the common vertex? Several strategies are conceivable. One strategy could be to assign the label of the preceding (subsequent) edge to a vertex. But this is somehow "unnatural" and even inconsistent, since a traversal of the sequence from the beginning to the end would yield a different labeling of vertices than a reverse traversal. Another strategy could be to associate each vertex with the label that is equal to the maximum (minimum) of the labels of all edges bounded by the vertex (maximum/minimum label rule). Such a fixed decision can, however, lead to an undesired loss of information. Hence, our strategy is to let the answer to this issue open in the sense that we associate with a vertex the labels of all edges bounded by that vertex (label union rule).

Let $E_{f}(\Omega)=\left\{\tilde{\boldsymbol{e}}\left|\operatorname{supp}(\tilde{\boldsymbol{e}}) \in E(\Omega), \mu(\tilde{\boldsymbol{e}}) \subseteq \Lambda \backslash\left\{\alpha_{1}\right\},\right| \mu(\tilde{\boldsymbol{e}}) \mid \in\{1,2\}\right\}$ be the set of all fuzzy edges over $E(\Omega)$; that is, an edge carries one or two labels. We allow the extension of the phrase " $A$ bounds $B$ " for crisp entities $A$ and $B$ to " $\tilde{A}$ bounds $\tilde{B}$ " for fuzzy entities $\tilde{A}$ and $\tilde{B}$. A simple fuzzy line (an example is shown in Fig. 5a) is defined as an alternating sequence $l=\left\langle\tilde{p}_{1}, \tilde{e}_{1}, \tilde{p}_{2}, \ldots, \tilde{p}_{n-1}, \tilde{e}_{n-1}, \tilde{p}_{n}\right\rangle$ such that

(i) $\forall 1 \leqslant i \leqslant n: \tilde{p}_{i} \in V_{f}(\Omega), \forall 1 \leqslant i<n: \tilde{e}_{i} \in E_{f}(\Omega) \wedge\left|\mu\left(\tilde{e}_{i}\right)\right|=1$

(ii) $\forall 1<i<j<n: \operatorname{supp}\left(\tilde{p}_{i}\right) \neq \operatorname{supp}\left(\tilde{p}_{j}\right)$

(iii) $\forall 1 \leqslant i<n: \tilde{p}_{i}$ and $\tilde{p}_{i+1}$ bound $\tilde{e}_{i}$

(iv) $\forall 1 \leqslant i \leqslant n: \mu\left(\tilde{p}_{i}\right)=\left\{\mu^{!}(\tilde{e}) \mid \tilde{e} \in\left\{\tilde{e}_{1}, \ldots, \tilde{e}_{n-1}\right\}, \tilde{p}_{i}\right.$ bounds $\left.\tilde{e}\right\}$

Condition (i) only admits exactly one label for an edge of a line. Condition (ii) requires that all vertices except for the end points are disjoint. Hence, it allows at most one cycle $\left(\operatorname{supp}\left(\tilde{p}_{1}\right)=\right.$ $\left.\operatorname{supp}\left(\tilde{p}_{n}\right)\right)$ since cycles within the sequence violate the condition. Thus, we can conclude that $\forall 1 \leqslant i<j<n: \operatorname{supp}\left(\tilde{e}_{i}\right) \neq \operatorname{supp}\left(\tilde{e}_{j}\right)$ must hold. We do not have to explicitly prohibit proper intersections of edges or touching situations of vertices with the interior of edges, because these cases are automatically excluded by the definition of the grid partition as a cellular complex. Condition (iv) describes the application of the label union rule to each vertex. Each vertex obtains the labels of its two incident edges. An exception is the case where $l$ is not a cycle and $\tilde{p}_{1}$ only gets the label of $\tilde{e}_{1}$ and $\tilde{p}_{n}$ only gets the label of $\tilde{e}_{n-1}$.

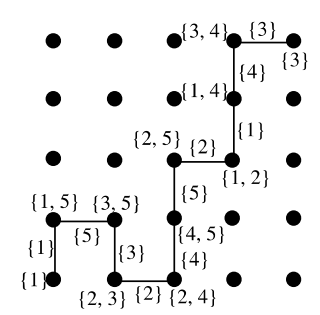

(a)

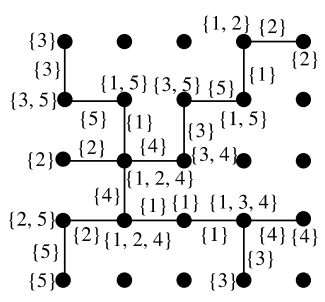

(b)

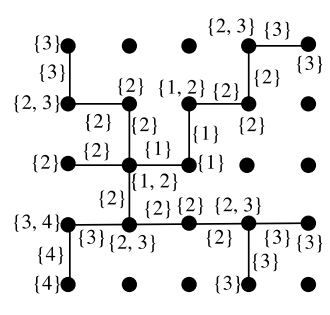

(c)

Fig. 5. Examples of a simple fuzzy line (a), a fuzzy line (b) and a smooth fuzzy line (c) (a label $i$ represents $\alpha_{i}$ ). 
For a simple fuzzy line $l$ we define the sets $V_{f}(l)=\left\{\widetilde{p}_{i} \mid 1 \leqslant i \leqslant n\right\}, E_{f}(l)=\left\{\widetilde{e}_{i} \mid 1 \leqslant i<n\right\}$, and $P_{f}(l)=\left\{\tilde{p}_{1}, \tilde{p}_{n}\right\}$. Let $V(l), E(l)$, and $P(l)$ be the sets containing the supports of the elements of $V_{f}(l), E_{f}(l)$, and $P_{f}(l)$, respectively, and let $S_{f}(\Omega)$ be the set of all simple fuzzy lines over $V(\Omega)$. A fuzzy block is a finite set $L=\left\{l_{1}, \ldots, l_{n}\right\} \subseteq S_{f}(\Omega)$ such that

(i) $\forall 1 \leqslant i<j \leqslant n: E\left(l_{i}\right) \cap E\left(l_{j}\right)=\emptyset$

(ii) $\forall 1 \leqslant i<j \leqslant n:\left(V\left(l_{i}\right) \backslash P\left(l_{i}\right)\right) \cap\left(V\left(l_{j}\right) \backslash P\left(l_{j}\right)\right)=\emptyset$

(iii) $\forall 1 \leqslant i \leqslant n \exists 1 \leqslant j \leqslant n, j \neq i: P\left(l_{i}\right) \cap P\left(l_{j}\right) \neq \emptyset$

(iv) $\forall \tilde{p} \in \bigcup_{1 \leqslant i \leqslant n} P_{f}\left(l_{i}\right): \mid\left\{\tilde{e} \in E_{f}\left(l_{i}\right) \mid 1 \leqslant i \leqslant n, \tilde{p}\right.$ bounds $\left.\tilde{e}\right\} \mid \neq 2$

(v) $\forall \tilde{p} \in \bigcup_{1 \leqslant i \leqslant n} P_{f}\left(l_{i}\right): \mu_{L}(\tilde{p})=\left\{\mu_{L}^{!}(\tilde{e}) \mid \exists 1 \leqslant i \leqslant n: \tilde{e} \in E_{f}\left(l_{i}\right) \wedge \tilde{p}\right.$ bounds $\left.\tilde{e}\right\}$

Conditions (i) and (ii) require that two distinct simple lines do not have common edges and common points (except for possible common end points). Condition (iii) ensures the property of connectedness of a block; isolated crisp simple lines are disallowed except for the case that the block consists exactly of one single line. Condition (iv) expresses that each end point of an element of $L$ must belong to exactly one or more than two bounded edges of $L$. This condition supports the requirement of maximal elements and hence achieves minimality of representation. Condition (v) expresses the label union rule for each end point of a simple fuzzy line.

All conditions together define a block as a maximum connected planar graph. The corresponding set of vertices is $V_{f}(L)=\bigcup\left\{V_{f}\left(l_{i}\right) \mid 1 \leqslant i \leqslant n\right\}$, and the set of edges is $E_{f}(L)=\bigcup\left\{E_{f}\left(l_{i}\right) \mid 1 \leqslant i \leqslant n\right\}$. Moreover, let $V(L)=\left\{\operatorname{supp}(\tilde{p}) \mid \tilde{p} \in V_{f}(L)\right\}$ and $E(L)=\left\{\operatorname{supp}(\tilde{e}) \mid \tilde{e} \in E_{f}(L)\right\}$. The set of all blocks over $S_{f}(\Omega)$ is denoted by $B_{f}(\Omega)$. The disjointedness of any two blocks $L_{1}, L_{2} \in B_{f}(\Omega)$ is defined as follows:

$$
L_{1} \text { and } L_{2} \text { are disjoint }: \Longleftrightarrow V\left(L_{1}\right) \cap V\left(L_{2}\right)=\emptyset \wedge E\left(L_{1}\right) \cap E\left(L_{2}\right)=\emptyset
$$

The "structured view" of a fuzzy spatial data type for discrete fuzzy lines (for an example see Fig. 5b) called fline is based on fuzzy blocks and is defined as follows:

$$
\text { fline }=\left\{D \subseteq B_{f}(\Omega) \mid \forall L_{1}, L_{2} \in D: L_{1} \text { and } L_{2} \text { are disjoint }\right\}
$$

The "flat view" emphasizes fuzzy points and fuzzy edges as the basic components of fuzzy lines:

$$
\text { fline }=\left\{Q_{1} \cup Q_{2} \mid Q_{1} \subseteq V_{f}(\Omega), Q_{2} \subseteq E_{f}(\Omega), \exists D \subseteq B_{f}(\Omega): Q_{1}=\bigcup_{L \in D} V_{f}(L), Q_{2}=\bigcup_{L \in D} E_{f}(L)\right\}
$$

We can also simply define the type line for discrete crisp lines as:

$$
\text { line }=\left\{L \in \text { fline }\left|\forall \tilde{e} \in E_{f}(L): \mu^{!}(\tilde{e})=1, \forall \tilde{p} \in V_{f}(L):\right| \mu(\tilde{p}) \mid=1, \mu^{!}(\tilde{p})=1\right\}
$$

Last but not least, we can give a definition for a special subtype of discrete fuzzy lines. This type called sfline (for smooth fuzzy lines) (Fig. 5c) takes into account "smooth" (that is, gradual) transitions within the interior of a line and models a kind of stepwise continuation ("discrete continuity"):

$$
\text { sfline }=\left\{L \in \text { fline }\left|\forall \tilde{p} \in V_{f}(L) \forall \alpha_{i}, \alpha_{j} \in \mu(\tilde{p}):\right| i-j \mid \in\{0,1\}\right\}
$$


This means that each point has either only one label or two neighbored labels in the ordered sequence of labels of $\Lambda$. This strategy models stepwise continuation.

Obviously, line $\subset$ sfline $\subset$ fline holds.

\subsection{Crisp and fuzzy regions}

The aim of this section is to develop and formalize a concept of discrete crisp and fuzzy regions. Our main interest concerns a structured view of discrete fuzzy regions. There are at least three possible, related interpretations for the membership of a grid unit in a fuzzy region. First, it may be interpreted as the degree of belonging to which the grid unit is inside or part of some areal feature. Consider the transition between a mountain and a valley and the problem to decide which grid units have to be assigned to the valley and which grid units to the mountain. Obviously, there is no strict boundary between them, and it seems to be more appropriate to model the transition by partial and multiple membership. Second, it may indicate the degree of compatibility of the grid unit with the attribute or concept represented by the fuzzy region. An example are "warm areas" where we must decide for each grid unit whether and to which grade it corresponds to the concept "warm". Third, it may be viewed as the degree of concentration of some attribute associated with the fuzzy region at the particular grid unit. An example is air pollution where we can assume the highest concentration at power stations, for instance, and lower concentrations with increasing distance from them. All these related interpretations give evidence of fuzziness.

We now start with the definition of a fuzzy grid unit. For this purpose we have to appropriately assign labels to its 2-cell, its four 1-cells, and its four 0-cells. The membership value of a grid unit as a whole is dominated by the 2-cell so that all its 0 - and 1-cells obtain this value.

A fuzzy grid unit for a point $p \in V(\Omega)$ and a label $\left\{\alpha_{k}\right\}$ is a triple $G_{f}^{k}(p)=\left(C_{f}^{k}(p), E_{f}^{k}(p), V_{f}^{k}(p)\right)$ such that $C_{f}^{k}(p)=C(p) \times \Lambda^{k, 1}, E_{f}^{k}(p)=E(p) \times \Lambda^{k, 2}$, and $V_{f}^{k}(p)=V(p) \times \Lambda^{k, 4}$ for some $1 \leqslant k \leqslant m$. That is, the 2-cell of a fuzzy grid unit is marked with a singelton label set (namely $\alpha_{k}$ ), each 1-cell with a set of up to two labels, and each 0 -cell with a set of up to four labels. Note that we also permit $\alpha_{1}=0$ as part of a label. As the only element of a label set it indicates the non-existence of a component of a fuzzy grid unit; in connection with other labels it shows the adjacency of such a component to the exterior of a fuzzy region. Due to $m$ possible $\alpha$-labels, for each point $p \in V(\Omega)$ we can define $m$ different fuzzy grid units (including a non-existing unit) which we collect in $G_{f}(p)=\bigcup_{k=1}^{m}\left\{G_{f}^{k}(p)\right\}$. We can also gather all possible 2-cells of a fuzzy grid unit for a point $p$ in $C_{f}(p)=\bigcup_{k=1}^{m} C_{f}^{k}(p)$, all possible 1-cells in $E_{f}(p)=\bigcup_{k=1}^{m} E_{f}^{k}(p)$, and all possible 0-cells in $V_{f}(p)=\bigcup_{k=1}^{m} V_{f}^{k}(p)$. Finally, the set of all fuzzy grid units over $V(\Omega)$ is $G_{f}(\Omega)=\bigcup_{p \in V(\Omega)} G_{f}(p)$, the set of all fuzzy unit areas is $C_{f}(\Omega)=\bigcup_{p \in V(\Omega)} C_{f}(p)$, the set of all fuzzy edges is $E_{f}(\Omega)=$ $\bigcup_{p \in V(\Omega)} E_{f}(p)$, and the set of all fuzzy vertices is $V_{f}(\Omega)=\bigcup_{p \in V(\Omega)} V_{f}(p)$.

Let $u_{1}=G_{f}^{k}(p), u_{2}=G_{f}^{l}(q) \in G_{f}(\Omega)$ for some $1<k, l \leqslant m$. We define the following topological predicates on fuzzy grid units:

(i) $u_{1}=u_{2} \quad: \Longleftrightarrow C(p)=C(q) \wedge E(p)=E(q) \wedge V(p)=V(q)$

(i) $u_{1}$ and $u_{2}$ 0-meet $: \Longleftrightarrow C(p) \cap C(q)=\emptyset \wedge E(p) \cap E(q)=\emptyset \wedge V(p) \cap V(q) \neq \emptyset$

(ii) $u_{1}$ and $u_{2} 1$-meet $: \Longleftrightarrow C(p) \cap C(q)=\emptyset \wedge E(p) \cap E(q) \neq \emptyset \wedge V(p) \cap V(q) \neq \emptyset$ 


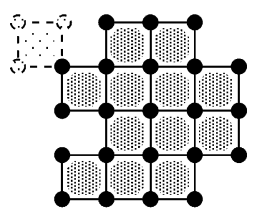

(a)

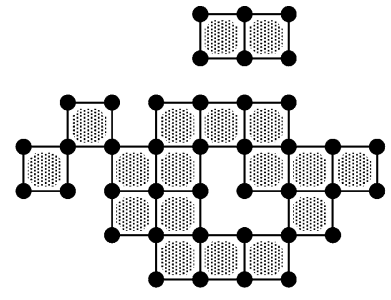

(b)

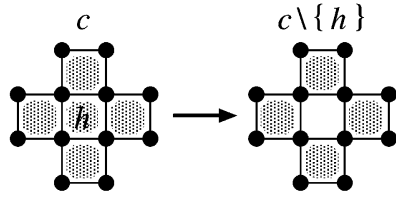

(c)

Fig. 6. An example of a fuzzy grid complex is shown in (a). Adding the dotted grid unit would invalidate the complex. Four faces are shown in (b). The example in (c) shows a simple situation where $c$ and $h$ are complexes but $c \backslash\{h\}$ is not a fuzzy face.

(iii) $u_{1}$ and $u_{2}$ are area-disjoint $: \Longleftrightarrow C(p) \cap C(q)=\emptyset$

(iv) $u_{1}$ and $u_{2}$ are edge-disjoint $: \Longleftrightarrow C(p) \cap C(q)=\emptyset \wedge E(p) \cap E(q)=\emptyset$

As the definitions show, these predicates are independent of the labels of a fuzzy grid unit. The predicate 0-meet implies that both fuzzy grid units have a zero-dimensional vertex in common while the predicate 1-meet implies that both fuzzy grid units meet in a one-dimensional edge and its two bounding vertices. The predicate area-disjoint allows both units to share a common edge together with the two bounding vertices whereas the predicate edge-disjoint only allows them to share a common vertex.

A fuzzy grid unit complex (an example is given in Fig. 6a) is a connected set of fuzzy grid units $c=\left\{u_{1}, \ldots, u_{n}\right\} \subseteq G_{f}(\Omega)$ with $u_{i}=G_{f}^{k_{i}}\left(p_{i}\right), p_{i} \in V(\Omega), 1<k_{i} \leqslant m$ such that

(i) $\forall 1 \leqslant i<j \leqslant n: u_{i}$ and $u_{j}$ are area-disjoint

(ii) $\forall 1 \leqslant i \leqslant n \exists 1 \leqslant j \leqslant n, j \neq i: u_{i}$ and $u_{j} 1$-meet

(iii) The point set $\partial \bigcup_{i=1}^{n}\left(p n t s\left(C\left(p_{i}\right)\right) \cup\right.$ pnts $\left(E\left(p_{i}\right)\right) \cup \operatorname{pnts}\left(V\left(p_{i}\right)\right)$ is a simple polygon.

(iv) $\forall 1 \leqslant i<j \leqslant n$ : $\left(u_{i}\right.$ and $u_{j} 1$-meet $\left.\wedge E\left(p_{i}\right) \cap E\left(p_{j}\right)=\{e\} \wedge V\left(p_{i}\right) \cap V\left(p_{j}\right)=\left\{q_{1}, q_{2}\right\}\right) \Rightarrow(\mu(\tilde{e}):=$ $\mu\left(C_{f}^{k_{i}}\left(p_{i}\right)\right) \cup \mu_{f}^{k_{j}}\left(C\left(p_{j}\right)\right), \mu\left(\tilde{q}_{1}\right):=\mu\left(\tilde{q}_{1}\right) \cup \mu\left(C_{f}^{k_{i}}\left(p_{i}\right)\right) \cup \mu\left(C_{f}^{k_{j}}\left(p_{j}\right)\right), \mu\left(\tilde{q}_{2}\right):=\mu\left(\tilde{q}_{2}\right) \cup \mu\left(C_{f}^{k_{i}}\left(p_{i}\right)\right) \cup$ $\left.\mu\left(C_{f}^{k_{j}}\left(p_{j}\right)\right)\right)$

(v) $\forall 1 \leqslant i<j \leqslant n: \quad\left(u_{i}\right.$ and $\quad u_{j} \quad 0$-meet $\left.\wedge V\left(p_{i}\right) \cap V\left(p_{j}\right)=\{q\}\right) \Rightarrow \mu(\tilde{q}):=\mu(\tilde{q}) \cup \mu_{f}^{k_{i}}\left(C\left(p_{i}\right)\right) \cup$ $\left.\mu\left(C_{f}^{k_{j}}\left(p_{j}\right)\right)\right)$

(vi) Let $\bar{c}=\left\{v_{1}, \ldots, v_{t}\right\}=G_{f}(\Omega) \backslash c \quad$ with $\quad v_{j}=G_{f}^{l}\left(q_{j}\right), q_{j} \in \Omega, 1 \leqslant l \leqslant m . \quad \forall 1 \leqslant i \leqslant n \forall 1 \leqslant j \leqslant$ $t:\left(u_{i}\right.$ and $v_{j} 1$-meet $\left.\wedge E\left(p_{i}\right) \cap E\left(q_{j}\right)=\{e\} \wedge V\left(p_{i}\right) \cap V\left(q_{j}\right)=\left\{s_{1}, s_{2}\right\}\right) \Rightarrow\left(\mu(\tilde{e}):=\mu\left(C_{f}^{k_{i}}\left(p_{i}\right)\right) \cup\right.$ $\left.\left\{\alpha_{1}\right\}, \mu\left(\tilde{s}_{1}\right):=\mu\left(\tilde{s}_{1}\right) \cup \mu\left(C_{f}^{k_{i}}\left(p_{i}\right)\right) \cup\left\{\alpha_{1}\right\}, \mu\left(\tilde{s}_{2}\right):=\mu\left(\tilde{s}_{2}\right) \cup \mu\left(C_{f}^{k_{i}}\left(p_{i}\right)\right) \cup\left\{\alpha_{1}\right\}\right)$

(vii) Let $\bar{c}=\left\{v_{1}, \ldots, v_{t}\right\}=G_{f}(\Omega) \backslash c$ with $v_{j}=G_{f}^{l}\left(q_{j}\right), q_{j} \in \Omega, \quad 1 \leqslant l \leqslant m . \forall 1 \leqslant i \leqslant n \forall 1 \leqslant j \leqslant$ $t:\left(u_{i}\right.$ and $v_{j} 0$-meet $\left.\wedge V\left(p_{i}\right) \cap V\left(q_{j}\right)=\{s\}\right) \Rightarrow \mu(\tilde{s}):=\mu(\tilde{s}) \cup \mu\left(C_{f}^{k_{i}}\left(p_{i}\right)\right) \cup\left\{\alpha_{1}\right\}$

Conditions (i) and (ii) formulate the partition character and the connectivity property, respectively. Condition (iii) prohibits holes in the complex. Condition (iv) applies the label union rule to all common edges and vertices of neighbored grid units. Each edge has one or two labels. It has two labels if the labels of the units are different; otherwise it has one label. The bounding vertices 
of the edge obtain the labels of the edge additionally to the labels they already have from other bounded edges; they can have at most four labels. Condition (v) appropriately labels the vertex of two grid units that 0 -meet. Conditions (vi) and (vii) deal with all vertices and edges that bound grid units of $c$ and that also belong to the complement of $c$; their label sets especially obtain the label $\alpha_{1}=0$ indicating their connection to the exterior. Edges of this kind have exactly two labels; vertices of this kind can have up to four labels. We call the conditions (iv) to (vii) label correction.

Let $C O_{f}(\Omega)=\left\{c \subseteq G_{f}(\Omega) \mid c\right.$ is a fuzzy grid unit complex $\}$, and let $c_{1}=\left\{u_{1}, \ldots, u_{n}\right\}, c_{2}=$ $\left\{v_{1}, \ldots, v_{t}\right\} \in C O_{f}(\Omega)$ with $u_{i}=G_{f}^{k_{i}}\left(p_{i}\right), p_{i} \in \Omega$, and $v_{j}=G_{f}^{l_{j}}\left(q_{j}\right), q_{j} \in \Omega, 1<k_{i}, l_{j} \leqslant m$. We define the following two predicates:

(i) $c_{1}$ and $c_{2}$ are edge-disjoint $: \Longleftrightarrow \forall 1 \leqslant i \leqslant n \forall 1 \leqslant j \leqslant t: u_{i}$ and $v_{j}$ are edge-disjoint

(ii) $c_{1}$ edge-inside $c_{2}: \Longleftrightarrow \forall 1 \leqslant i \leqslant n \exists 1 \leqslant j \leqslant t: p_{i}=q_{j} \wedge\left(\forall \tilde{e} \in E_{f}^{l_{j}}\left(q_{j}\right): \alpha_{1}=0 \notin \mu(\tilde{e})\right)$

The predicate edge-disjoint implies that two fuzzy grid unit complexes may only share single vertices but no edges. Otherwise, we could merge them together into a single fuzzy grid unit complex. The predicate edge-inside first checks whether each grid unit of $c_{1}$ is contained in $c_{2}$ and whether the label of the corresponding 2-cell of $c_{1}$ is not greater than the label of the corresponding 2-cell of $c_{2}$. Another condition additionally requires that the label sets of the edges of all common fuzzy grid units of $c_{1}$ and $c_{2}$ do not contain label 0 for $c_{2}$. This, in particular, means that the vertices of these grid units are allowed to have label 0 in $c_{2}$. Altogether, this condition identifies the corresponding grid units of $c_{1}$ in $c_{2}$ and tests whether $c_{2}$ 's edges contain label 0 . If this is not the case, then we know that $c_{1}$ lies properly in $c_{2}$, otherwise $c_{1}$ contains at least one grid unit lying on the "thick boundary" of a grid unit in $c_{2}$. Again both topological predicates are defined irrespective of labels.

A fuzzy face $f$ (see Fig. 6b) is a pair $(c, H)$ with $c \in C O_{f}(\Omega), H=\left\{h_{1}, \ldots, h_{n}\right\}$ with $h_{i} \in C O_{f}(\Omega)$ such that the following conditions hold (let $U_{f}(H)=\bigcup_{1 \leqslant i \leqslant n} h_{i}$, let $p, q \in V(\Omega), 1<k, l \leqslant m$, and let $U_{f}(f)$ denote the set of all grid units of $f$ ):

(i) $\forall u \in U_{f}(H): u=G_{f}^{k}(p) \Rightarrow \mu_{u}^{!}(C(p))=\alpha_{m}=1$ (or: $k=m$ )

(ii) $\forall u \in U_{f}(H) \exists v \in c: u=G_{f}^{k}(p) \wedge v=G_{f}^{l}(q) \wedge p=q \wedge \mu_{v}^{!}(C(q))=\mu_{u}^{!}(C(p))$

(iii) $\forall 1 \leqslant i \leqslant n: h_{i}$ edge-inside $c$

(iv) $\forall 1 \leqslant i<j \leqslant n: h_{i}$ and $h_{j}$ are edge-disjoint

(v) $U_{f}(f)=c \backslash U_{f}(H)$

(vi) Label correction applied to $U_{f}(F)$.

(vii) $\forall u \in U_{f}(f) \exists v \in U_{f}(f), u \neq v: u$ and $v 1$-meet

Conditions (i) and (ii) address the problem of defining a concept of "fuzzy holes" (see also the discussion in [23]). In fact, there are only crisp holes, since only they can express parts that are enclosed by a fuzzy grid unit complex and that do definitely not lie within the interior of $c$. We therefore have to conceptually require that the units composing the holes are crisp in $c$, too. Condition (iii) requires that all holes are edge-inside $c$. A hole is only allowed to share vertices and not edges with the exterior of $c$; otherwise we would form a "bay" in $c$, and we should have omitted the hole unit from the structure definition of $c$ before. Condition (iv) requires that any two holes share at most vertices and not edges; otherwise we could merge them together into one hole. Condition (v) describes the set of units belonging to $f$. Unfortunately, the set difference can 
yield a collection of fuzzy grid units which is not connected (see Fig. 6c). The connectivity property is required in condition (vii).

Let $F_{f}(\Omega)$ denote the set of all fuzzy faces over $V(\Omega)$, and let $f=\left(f_{0}, \bar{F}\right), g=\left(g_{0}, \bar{G}\right) \in F_{f}(\Omega)$. We can then define the predicate:

$f$ and $g$ are edge-disjoint $: \Longleftrightarrow f_{0}$ and $g_{0}$ are edge-disjoint $\vee \exists \bar{g} \in \bar{G}: f_{0}$ edge-inside $\bar{g} \vee \exists \bar{f}$ $\in \bar{F}: g_{0}$ edge-inside $\bar{f}$

The "structured view" of a spatial data type for discrete fuzzy regions called fregion is based on fuzzy faces and is defined as follows:

$$
\text { fregion }=\left\{F \subseteq F_{f}(\Omega) \mid \forall f, g \in F: f \text { and } g \text { are edge-disjoint }\right\}
$$

The "flat view" emphasizes fuzzy grid units as the basic component of fuzzy regions:

$$
\text { fregion }=\left\{U \subseteq G_{f}(\Omega) \mid \exists F \subseteq F_{f}(\Omega): U=\bigcup_{f \in F} U_{f}(f)\right\}
$$

Given $F \in$ fregion, let $U_{f}(F)=\bigcup_{f \in F} U_{f}(f)$ be the set of all fuzzy grid units of $F$. We can then simply define the type region for discrete crisp regions as:

$$
\text { region }=\left\{F \in \text { fregion } \mid \forall u=G_{f}^{k}(p) \in U_{f}(F), p \in V(\Omega), 1<k \leqslant m: \mu_{u}^{\prime}(C(p))=1\right\}
$$

Moreover, we define $C_{f}(F)=\left\{\tilde{c} \in C_{f}(\Omega) \mid \exists u=G_{f}^{k}(p) \in U_{f}(F), p \in V(\Omega), 1<k \leqslant m: \tilde{c}=C_{f}^{k}(p)\right\}$ as the set of all fuzzy unit areas, $E_{f}(F)=\left\{\tilde{e} \in E_{f}(\Omega) \mid \exists u \in U_{f}(F): \tilde{e}\right.$ bounds $\left.u\right\}$ as the set of all fuzzy edges and $V_{f}(F)=\left\{\tilde{p} \in V_{f}(\Omega) \mid \exists u \in U_{f}(F): \tilde{p}\right.$ bounds $\left.u\right\}$ as the set of all fuzzy points of $F$.

Finally, we can give a definition for a special subtype of discrete fuzzy regions. This type called sfregion (for smooth fuzzy regions) takes into account "smooth" (that is, gradual) transitions within the interior of a region and thus models a kind of stepwise continuation ("discrete continuity"):

$$
\begin{aligned}
\text { sfregion }= & \left\{F \in \text { fregion } \mid \forall u=G_{f}^{k}(p), v=G_{f}^{l}(q) \in U_{f}(F), p, q \in V(\Omega), 1<k,\right. \\
& l \leqslant m: u \text { and } v 1 \text {-meet } \Rightarrow|k-l| \leqslant 1\}
\end{aligned}
$$

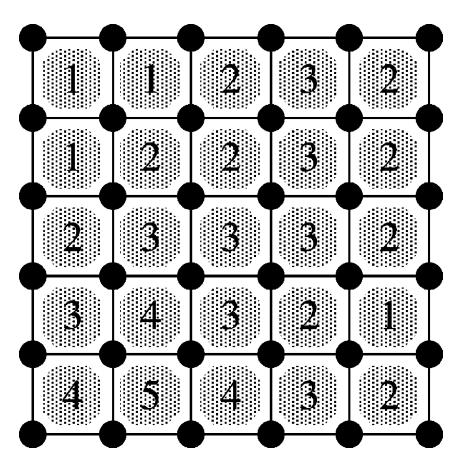

Fig. 7. An example of the labeling strategy in a smooth fuzzy region. The label $i$ stands for $\alpha_{i}$, and only the labels of 2-cells are shown. 


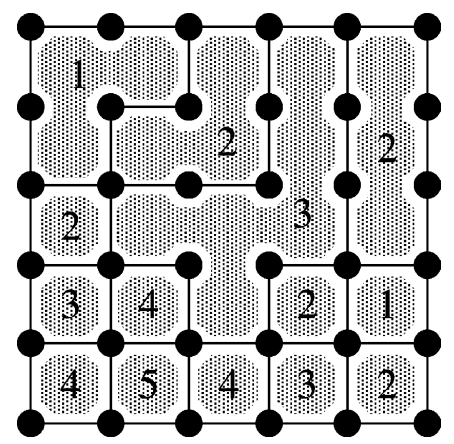

Fig. 8. Transformation of the representation of a fuzzy region based on grid partitions to a fuzzy region based on spatial partitions.

This means that two adjacent grid units have either the same label or two neighbored labels in the ordered sequence of labels of $\Lambda$. This strategy models stepwise continuation. Fig. 7 shows a schematic example.

The grid units of a fuzzy region that carry the same label form a subregion of the fuzzy region. In a vector-oriented setting, for reasons of efficient representation, we can merge adjacent grid units carrying the same label for their 2-cells. This leads from a grid partition to a spatial partition in the sense of [12] and from the representation in Fig. 7 to the representation in Fig. 8.

Last but not least we can obviously conclude that region $\subset$ sfregion $\subset$ fregion holds.

\section{Geometric union, intersection, and difference}

For each of the three spatial data types we will now first define the three geometric operations

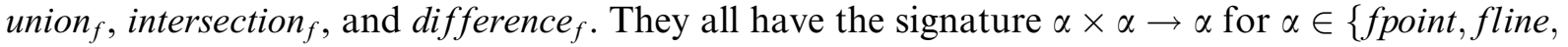
fregion $\}$, and hence the three data types are to be closed under these operations. Let $P, Q \in$ fpoint. For the first two geometric operations we can employ the union and intersection operations on fuzzy sets (see Section 4.1) and define union $_{f}(P, Q)=P \cup Q$ and intersection $(P, Q)=P \cap Q$. The union (intersection) operation takes the maximum (minimum) membership value for each point of $V(\Omega)$ with respect to its degree of belonging to $P$ and $Q$. The meaning of geometric difference is not defined with the aid of the difference on fuzzy sets (that is, difference $_{f}(P, Q) \neq P \cap \neg Q$ ), since the right side of the inequality does not make great sense in the spatial context. Instead, we define:

$$
\begin{aligned}
\text { difference }_{f}(P, Q)=\{ & \left((x, y), \mu_{\text {difference }_{f}(P, Q)}((x, y))\right) \mid(x, y) \in V(\Omega), \mu_{\text {difference }_{f}(P, Q)}^{!}((x, y)) \\
= & \text { if } \mu_{P}^{!}((x, y)) \leqslant \mu_{Q}^{\prime}((x, y)) \text { then } 0 \\
& \left.\quad \text { else } \min \left\{\alpha_{i} \in \Lambda \mid \alpha_{i} \geqslant \mu_{P}^{\prime}((x, y))-\mu_{Q}^{!}((x, y))\right\}\right\}
\end{aligned}
$$

The idea is that the membership value of a point is diminished by the membership value of another point having the same coordinates. In the crisp case this leads to a total elimination of the first point and in the fuzzy case to a partial elimination of the first point. 
For the geometric operations on fuzzy lines we consider their edge and vertex sets, that is, we take the flat view. Let $L_{1}, L_{2} \in$ fline. The union operation is then defined on the basis of the union operation on fuzzy sets as follows:

$$
\begin{aligned}
\text { union }_{f}\left(L_{1}, L_{2}\right)=\{ & L \in \text { fline } \mid E(L)=E\left(L_{1}\right) \cup E\left(L_{2}\right), V(L)=\left\{\tilde{p} \in V_{f}(\Omega) \mid \exists \tilde{e}\right. \\
& \left.\left.\in E(L): \tilde{p} \text { bounds } \tilde{e}, \mu(\tilde{p})=\left\{\mu^{!}(\tilde{e}) \mid \tilde{e} \in E(L), \tilde{p} \text { bounds } \tilde{e}\right\}\right\}\right\}
\end{aligned}
$$

That is, if $L_{1}$ and $L_{2}$ have an edge in common, then it is part of the union and obtains the maximum label of both edges in $L_{1}$ and $L_{2}$. All edges of $L_{1}\left(L_{2}\right)$ whose support is not contained in $L_{2}\left(L_{1}\right)$ are simply added unchanged to the result. The labeling of all vertices bounding edges is updated correspondingly. The intersection operation is defined analogously with fuzzy intersection. The difference operation pursues the same labeling policy as the difference operation on fuzzy points; its detailed definition is omitted here.

The definitions of the geometric operations on fuzzy regions are also based on the flat view. In case of union $_{f}$ we gather all grid units contained in the two fuzzy regions, label common 2-cells of the grid units with the maximum membership value, and adjust the label sets of bounding fuzzy vertices and edges according to the label correction mechanism. Let $F_{1}, F_{2}, F \in$ fregion. The union operation is then defined on the basis of the union operation on fuzzy sets, and we obtain $F=$ union $_{f}\left(F_{1}, F_{2}\right)$ such that

(i) $\forall p \in V(\Omega) \forall 1<k, l \leqslant m$ :

$$
\begin{aligned}
& \left(\left(\exists s=G_{f}^{k}(p) \in U_{f}\left(F_{1}\right) \wedge \exists t=G_{f}^{l}(p) \in U_{f}\left(F_{2}\right)\right) \Rightarrow \exists u=G_{f}^{\max (k, l)}(p) \in U_{f}(F)\right) \vee \\
& \left(\left(\exists s=G_{f}^{k}(p) \in U_{f}\left(F_{1}\right) \wedge \nexists t=G_{f}^{l}(p) \in U_{f}\left(F_{2}\right)\right) \Rightarrow \exists u=G_{f}^{k}(p) \in U_{f}(F)\right) \vee \\
& \left(\left(\nexists s=G_{f}^{k}(p) \in U_{f}\left(F_{1}\right) \wedge \exists t=G_{f}^{l}(p) \in U_{f}\left(F_{2}\right)\right) \Rightarrow \exists u=G_{f}^{l}(p) \in U_{f}(F)\right)
\end{aligned}
$$

(ii) Label correction applied to $U_{f}(F)$.

Correspondingly, the operation intersection $_{f}\left(F_{1}, F_{2}\right)$ rests on the intersection of fuzzy sets and assigns the minimum membership value to fuzzy grid units that are part of both regions. Hence, we replace condition (i) from above with

(i) $\forall p \in V(\Omega) \forall 1<k, l \leqslant m$ :

$$
\left(\exists s=G_{f}^{k}(p) \in U_{f}\left(F_{1}\right) \wedge \exists t=G_{f}^{l}(p) \in U_{f}\left(F_{2}\right)\right) \Rightarrow \exists u=G_{f}^{\min (k, l)}(p) \in U_{f}(F)
$$

The meaning of the operation difference $_{f}$ is also not defined with the aid of the difference on fuzzy sets. Instead we replace condition (i) from above with

(i) $\forall p \in V(\Omega) \forall 1<k, l \leqslant m$ :

$$
\begin{aligned}
& \left(\left(\exists s=G_{f}^{k}(p) \in U_{f}\left(F_{1}\right) \wedge \nexists t=G_{f}^{l}(p) \in U_{f}\left(F_{2}\right)\right) \Rightarrow \exists u=G_{f}^{k}(p) \in U_{f}(F)\right) \vee \\
& \left(\left(\exists s=G_{f}^{k}(p) \in U_{f}\left(F_{1}\right) \wedge \exists t=G_{f}^{l}(p) \in U_{f}\left(F_{2}\right) \wedge k>l\right) \Rightarrow\right. \\
& \left.\left(\exists u=G_{f}^{w}(p) \in U_{f}(F) \wedge w=\min \left\{v \in\{2, \ldots, m\} \mid \alpha_{v} \geqslant \alpha_{k}-\alpha_{l}\right\}\right)\right) \vee \\
& \left(\left(\exists s=G_{f}^{k}(p) \in U_{f}\left(F_{1}\right) \wedge \exists t=G_{f}^{l}(p) \in U_{f}\left(F_{2}\right) \wedge k \leqslant l\right) \Rightarrow\left(\exists u=G_{f}^{1}(p) \notin U_{f}(F)\right)\right)
\end{aligned}
$$


In the end we present three mixed operations. The first (overloaded) operation has the signature intersection $_{f}:$ fline $\times$ fline $\rightarrow$ fpoint and computes all fuzzy points resulting from the intersection of two fuzzy lines. Let $L_{1}, L_{2} \in$ fline.

$$
\begin{aligned}
\text { intersection }_{f}\left(L_{1}, L_{2}\right)= & \left\{\tilde{p} \in f p o i n t \mid \exists \tilde{p}_{1} \in V_{f}\left(L_{1}\right) \exists \tilde{p}_{2} \in V_{f}\left(L_{2}\right): \operatorname{supp}(\tilde{p})=\operatorname{supp}\left(\tilde{p}_{1}\right)\right. \\
& \left.=\operatorname{supp}\left(\tilde{p}_{2}\right), \mu(\tilde{p})=\min \left\{\mu\left(\tilde{p}_{1}\right), \mu\left(\tilde{p}_{2}\right)\right\}\right\}
\end{aligned}
$$

The second (overloaded) operation is intersection $f_{f}: \alpha \times \beta \rightarrow$ fline with $\alpha, \beta \in\{$ fline, fregion $\}$, $\alpha \neq \beta$. It computes the intersection of a fuzzy line and a fuzzy region. Let $L \in$ fline and $F \in$ fregion. Then intersection ${ }_{f}(L, F)=K$ such that

(i) $E_{f}(K)=\left\{\tilde{e} \in E_{f}(\Omega) \mid \exists \tilde{e}_{1} \in E_{f}(L) \exists \tilde{e}_{2} \in E_{f}(F): \operatorname{supp}(\tilde{e})=\operatorname{supp}\left(\tilde{e}_{1}\right)=\operatorname{supp}\left(\tilde{e}_{2}\right)\right.$, $\left.0 \notin \mu\left(\tilde{e}_{2}\right), \mu(\tilde{e})=\left\{\min \left(\mu\left(\tilde{e}_{1}\right), \min \left(\mu\left(\tilde{e}_{2}\right)\right)\right)\right\}\right\}$

(ii) $V_{f}(K)=\left\{\tilde{p} \in V_{f}(\Omega) \mid \exists \tilde{e} \in E_{f}(K): \tilde{p}\right.$ bounds $\left.\tilde{e}\right\}$,

$\mu_{K}(\tilde{p})=\left\{\mu^{!}(\tilde{e}) \mid \tilde{e} \in E_{f}(K), \tilde{p}\right.$ bounds $\left.\tilde{e}\right\}$

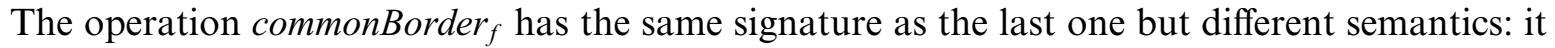
computes the common boundary parts of a fuzzy line and a fuzzy region. It also has the same definition as the last operation except for the condition $0 \notin \mu\left(\tilde{e}_{2}\right)$ in (i). Here we have to require that $0 \in \mu\left(\tilde{e}_{2}\right)$. The membership value 0 indicates that an an edge belongs to the boundary.

\section{Topological predicates}

In this section we deal with topological predicates for discrete crisp and fuzzy spatial objects. Predicates computing topological relationships between spatial objects are very important for spatial databases, GIS, and image databases. In particular, they are needed in spatial query languages where they are, for instance, employed as part of a filter condition in a query. For the Euclidean space, topological relationships have been studied very intensively. An important approach rests on the so-called 9-intersection model $[9,10]$ from which a canonical collection of topological relationships can be derived for each combination of spatial types. The model is based on the nine possible intersections of boundary $(\partial A)$, interior $\left(A^{\circ}\right)$, and exterior $\left(A^{-}\right)$of a spatial object $A$ with the corresponding components of another object $B$. Each intersection is tested with regard to the topologically invariant criteria of emptiness and non-emptiness. This can be expressed by evaluating the following matrix:

$$
\left(\begin{array}{lll}
\partial A \cap \partial B \neq \emptyset & \partial A \cap B^{\circ} \neq \emptyset & \partial A \cap B^{-} \neq \emptyset \\
A^{\circ} \cap \partial B \neq \emptyset & A^{\circ} \cap B^{\circ} \neq \emptyset & A^{\circ} \cap B^{-} \neq \emptyset \\
A^{-} \cap \partial B \neq \emptyset & A^{-} \cap B^{\circ} \neq \emptyset & A^{-} \cap B^{-} \neq \emptyset
\end{array}\right)
$$

For this matrix $2^{9}=512$ different configurations are possible from which only a certain subset makes sense depending on the combination of spatial objects just considered. In this paper we will only deal with regions. A restriction of the 9-intersection model is that the regions considered must be homeomorphic to the closed disc, that is, they must be connected and are not allowed to have holes. We call this subtype of regions region', and if we speak of regions in this section, we relate to them in the restricted sense just described. For two regions, eight meaningful configu- 
rations have been identified which lead to the eight predicates called disjoint, meet, overlap, equal, inside, contains, covers, and coveredBy. Each predicate is associated with a unique intersection matrix so that all predicates are mutually exclusive and complete with regard to the topologically invariant criteria of emptiness and non-emptiness:

$$
\begin{aligned}
& \left(\begin{array}{lll}
0 & 0 & 1 \\
0 & 0 & 1 \\
1 & 1 & 1
\end{array}\right) \quad\left(\begin{array}{lll}
1 & 0 & 1 \\
0 & 0 & 1 \\
1 & 1 & 1
\end{array}\right) \quad\left(\begin{array}{lll}
1 & 1 & 1 \\
1 & 1 & 1 \\
1 & 1 & 1
\end{array}\right) \quad\left(\begin{array}{lll}
1 & 0 & 0 \\
0 & 1 & 0 \\
0 & 0 & 1
\end{array}\right) \\
& \text { disjoint } \\
& \text { meet } \\
& \text { overlap } \\
& \text { equal } \\
& \underset{\text { conside }}{\left(\begin{array}{lll}
0 & 1 & 0 \\
0 & 1 & 0 \\
1 & 1 & 1
\end{array}\right)} \underset{\text { contains }}{\left(\begin{array}{lll}
0 & 0 & 1 \\
1 & 1 & 1 \\
0 & 0 & 1
\end{array}\right)} \quad \underset{\left.\begin{array}{lll}
1 & 0 & 1 \\
1 & 1 & 1 \\
0 & 0 & 1
\end{array}\right)}{\text { covers }} \underbrace{\left(\begin{array}{lll}
1 & 1 & 0 \\
0 & 1 & 0 \\
1 & 1 & 1
\end{array}\right)}_{\text {coveredBy }}
\end{aligned}
$$

Since our crisp and fuzzy spatial objects are part of the grid partition and since the grid partition is embedded into the Euclidean space and is a topological space, the spatial objects (and the grid partition) enjoy the topological properties of $\mathbb{R}^{2}$. Hence, it is permissible to apply the 9-intersection model to discrete crisp spatial objects. Unfortunately, we do, so far, not have concepts of boundary, interior, and exterior in our model. Consequently, we have to investigate in which manner unit areas, edges, and vertices contribute to these three topological concepts.

Let $F \in$ region', and let $C(F)$ be the set of crisp unit areas, $E(F)$ be the set of crisp edges, and $V(F)$ be the set of crisp vertices of $F$, that is, all components carry the label $\{1\}$. We specify how interior, boundary, and exterior of $F$ can be expressed by them.

$$
\begin{array}{lll}
\mathscr{C}(\partial F)=\emptyset & \mathscr{C}\left(F^{\circ}\right)=C(F) & \mathscr{C}\left(F^{-}\right)=C(\Omega) \backslash C(F) \\
\mathscr{E}(\partial F)=\{e \in E(F) \mid 0 \in \mu(e)\} & \mathscr{E}\left(F^{\circ}\right)=E(F) \backslash \mathscr{E}(\partial F) & \mathscr{E}\left(F^{-}\right)=E(\Omega) \backslash E(F) \\
\mathscr{V}(\partial F)=\{p \in V(F) \mid 0 \in \mu(p)\} & \mathscr{V}\left(F^{\circ}\right)=V(F) \backslash \mathscr{V}(\partial F) & \mathscr{V}\left(F^{-}\right)=V(\Omega) \backslash V(F)
\end{array}
$$

This enables us now to describe the three topological sets of a crisp region $F$ occurring in the intersection matrix as:

$$
\begin{aligned}
& \partial F=\mathscr{C}(\partial F) \cup \mathscr{E}(\partial F) \cup \mathscr{V}(\partial F) \\
& F^{\circ}=\mathscr{C}\left(F^{\circ}\right) \cup \mathscr{E}\left(F^{\circ}\right) \cup \mathscr{V}\left(F^{\circ}\right) \\
& F^{-}=\mathscr{C}\left(F^{-}\right) \cup \mathscr{E}\left(F^{-}\right) \cup \mathscr{V}\left(F^{-}\right)
\end{aligned}
$$

These sets can now be used to compute the intersection sets in the matrix. We must, of course, pay attention to the fact that only sets of compatible types can be combined. For example, the interior-interior intersection between two regions $F$ and $G$ can and must be limited to the following computation:

$$
F^{\circ} \cap G^{\circ}=\left(\mathscr{C}\left(F^{\circ}\right) \cap \mathscr{C}\left(G^{\circ}\right)\right) \cup\left(\mathscr{E}\left(F^{\circ}\right) \cap \mathscr{E}\left(G^{\circ}\right)\right) \cup\left(\mathscr{V}\left(F^{\circ}\right) \cap \mathscr{V}\left(G^{\circ}\right)\right)
$$

This amounts to 27 intersection sets that apparently have to be calculated. But this number can be reduced to nine if we look at the possible dimensions of the entries in the intersection matrix. The observation (with one exception) is that the dimension of the intersection of two spatial components having dimension $n$ and $m$, respectively, is either equal to $\min (n, m)$, or the intersection is empty. The intersection of two open areas (interior or exterior) is two-dimensional or empty. This leads to four cases. The intersection of a boundary with an open area (interior or exterior) is 
one-dimensional or empty. This leads also to four cases. An exception is the boundary-boundary intersection which can have a one-dimensional (common edges) or a zero-dimensional (common vertices) result, or which can be empty. If this intersection has common edges, then this intersection has also common vertices, namely at least those which bound the intersecting edges. If the dimension of the intersection is zero, it is obvious that the intersection has no common edges. Hence, it must have common one-dimensional vertices. In summary, we can pose the following intersection matrix for discrete crisp regions:

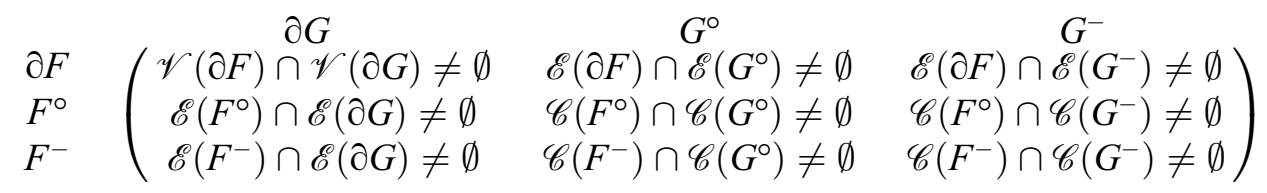

This intersection matrix can be the basis for a treatment of topological relationships between discrete fuzzy regions. The restriction to objects of type region' also leads to a restriction of fuzzy regions in the sense that we can here only permit simple fuzzy regions. A simple fuzzy region is a region that is $\alpha$-connected and where each $\alpha$-level region (see definition of an $\alpha$-set in Section 4.1) is an element of type region. A fuzzy region is called $\alpha$-connected if all its $\alpha$-level regions $F_{\alpha_{i}}$ for $\alpha_{i} \in \Lambda$ are simple crisp regions, that is, elements of region'. Since $\alpha_{i}<\alpha_{i+1}$, we obtain $F_{\alpha_{i}} \supseteq F_{\alpha_{i+1}}$ for $1 \leqslant i<n$, that is, the $\alpha$-level regions are nested. This describes some kind of a "concentric" model of a fuzzy region with its core in the center and more vague parts in the core's environment. With increasing distance from the center the degree of membership decreases.

The remaining question now is how to employ the $\alpha$-level regions for determining the topological relationships between two simple fuzzy regions. We use the concept of basic probability assignment [7] for this purpose. A basic probability assignment $m\left(F_{\alpha_{i}}\right)$ can be attached to each $\alpha$ level region $F_{\alpha_{i}}$ and can be interpreted as the probability that $F_{\alpha_{i}}$ is the "true" representative of $F$. It is defined as $m\left(F_{\alpha_{i}}\right)=\alpha_{i}-\alpha_{i-1}$ for $1<i \leqslant n$ with $\alpha_{1}=0$ and $\alpha_{n}=1$. It is easy to see that $\sum_{1<i \leqslant n} m\left(F_{\alpha_{i}}\right)=\alpha_{n}-\alpha_{1}=1-0=1$.

Let $\pi_{f}(F, G)$ be the value that represents a relationship $\pi_{f}$ between two simple fuzzy regions $F$ and $G$. Based on the work in [7] this relationship can be determined by

$$
\pi_{f}(F, G)=\sum_{i=2}^{n} \sum_{j=2}^{n} m\left(F_{\alpha_{i}}\right) \cdot m\left(G_{\alpha_{j}}\right) \cdot \pi_{c}\left(F_{\alpha_{i}}, G_{\alpha_{j}}\right)
$$

where $\pi_{c}\left(F_{\alpha_{i}}, G_{\alpha_{j}}\right) \in\{0,1\}$ checks the validity of predicate $\pi_{c}$ for two simple crisp $\alpha$-level regions $F_{\alpha_{i}}$ and $G_{\alpha_{j}}$. This formula is equivalent to:

$$
\pi_{f}(F, G)=\sum_{i=2}^{n} \sum_{j=2}^{n}\left(\alpha_{i}-\alpha_{i-1}\right) \cdot\left(\alpha_{j}-\alpha_{j-1}\right) \cdot \pi_{c}\left(F_{\alpha_{i}}, G_{\alpha_{j}}\right)
$$

If $\pi_{c}$ is one of our eight topological predicates out of $\{$ disjoint, meet, overlap, equal, inside, contains, covers, coveredBy\}, we can compute the degree of the corresponding topological relationship between two simple fuzzy regions, that is, $0 \leqslant \pi_{f}(F, G) \leqslant 1$. The value of $\pi_{c}\left(F_{\alpha_{i}}, G_{\alpha_{j}}\right)$ is either 1 (true) or 0 (false). Once this value has been determined for all combinations of $\alpha$-level regions from $F$ and $G$, the aggregated value of the topological predicate $\pi_{f}(F, G)$ can be computed as shown above. 
It remains to show that $0 \leqslant \pi_{f}(F, G) \leqslant 1$ actually holds, that is, $\pi_{f}$ is really a fuzzy predicate. Since $\alpha_{i}-\alpha_{i-1}>0$ for all $2 \leqslant i \leqslant n$ and since $\pi_{c}\left(F_{\alpha_{i}}, G_{\alpha_{j}}\right) \geqslant 0$ for all $2 \leqslant i, j \leqslant n, \pi_{f}(F, G) \geqslant 0$ holds. We can show the other inequality by determining an upper bound for $\pi_{f}(F, G)$ :

$$
\begin{aligned}
\pi_{f}(F, G)= & \sum_{i=2}^{n} \sum_{j=2}^{n}\left(\alpha_{i}-\alpha_{i-1}\right) \cdot\left(\alpha_{j}-\alpha_{j-1}\right) \cdot \pi_{c r}\left(F_{\alpha_{i}}, G_{\alpha_{j}}\right) \\
\leqslant & \sum_{i=2}^{n} \sum_{j=2}^{n}\left(\alpha_{i}-\alpha_{i-1}\right) \cdot\left(\alpha_{j}-\alpha_{j-1}\right) \quad\left(\text { since } \pi_{c}\left(F_{\alpha_{i}}, G_{\alpha_{j}}\right) \leqslant 1\right) \\
= & \left(\alpha_{2}-\alpha_{1}\right)\left(\alpha_{2}-\alpha_{1}\right) \\
& +\cdots+\left(\alpha_{2}-\alpha_{1}\right)\left(\alpha_{n}-\alpha_{n-1}\right)+\cdots+\left(\alpha_{n}-\alpha_{n-1}\right)\left(\alpha_{2}-\alpha_{1}\right) \\
& +\cdots+\left(\alpha_{n}-\alpha_{n-1}\right)\left(\alpha_{n}-\alpha_{n-1}\right) \\
= & \left(\alpha_{2}-\alpha_{1}\right)\left(\left(\alpha_{2}-\alpha_{1}\right)+\cdots+\left(\alpha_{n}-\alpha_{n-1}\right)\right) \\
& +\cdots+\left(\alpha_{n}-\alpha_{n-1}\right)\left(\left(\alpha_{2}-\alpha_{1}\right)+\cdots+\left(\alpha_{n}-\alpha_{n-1}\right)\right) \\
= & \left(\alpha_{2}-\alpha_{1}\right)+\cdots+\left(\alpha_{n}-\alpha_{n-1}\right)\left(\text { since } \sum_{i=2}^{n}\left(\alpha_{i}-\alpha_{i-1}\right)=1\right) \\
= & 1
\end{aligned}
$$

Hence, $\pi_{f}(F, G) \leqslant 1$ holds.

\section{Implementation}

In this section we describe how finite resolution crisp and fuzzy spatial objects can be implemented. Since we have incorporated the important aspect of finite representation explicitly into our spatial model and since we have used a bottom-up approach for the definition of our data types, it is sufficient to give an implementation strategy only for the basic components of our data model, namely for vertices, edges, unit areas, grid units, grid partitions, and their fuzzy counterparts. The implementation of the more complex components built upon the basic ones is then straightforward according to the definitions given in this paper.

As the underlying geometric domain for our objects we have selected a grid partition which itself is built upon a homogeneous grid given as the finite point set $\Omega=\{-n, \ldots, n\}^{2} \subset \mathbb{Z}^{2}$ with an arbitrary but fixed and representable $n \in \mathbb{N}$. In practice, the point coordinates can be directly represented by values of integer data types as they are available in programming languages, or by special, higher precision implementations of number systems (for example, arbitrarily long integers). Then a vertex is simply a point $p=(x, y) \in \Omega$. An edge can be represented as a lexicographically ordered pair $(p, q)$ with $p, q \in \Omega, p=(x, y), q=(x, y+1), y<n$, or $q=(x+1, y)$, $x<n$. A unit area is represented as a lexicographically ordered pair $(p, q)$ with $p, q \in \Omega, p=(x, y)$ and $q=(x+1, y+1), x<n, y<n$, where $p$ denotes the bottom left and $q$ the top right corner of the pertaining open rectangle. Actually, it would be sufficient to represent a unit area also by a point $(x, y) \in V(\Omega)$, because its extent is automatically given. 
A grid unit for a point $p=(x, y) \in \Omega$ is the triple $G(p)=(C(p), E(p), V(p))$ such that $C(p)=$ $\{(p, q)\}$ with $p, q \in \Omega, p=(x, y), q=(x+1, y+1), x<n, y<n$, is the unit area, $V(p)=$ $\left\{p_{0}, \ldots, p_{3}\right\}$ with $p_{0}=p, p_{1}=(x+1, y), p_{2}=(x+1, y+1)$, and $p_{3}=(x, y+1)$ are the bounding vertices, and $E(p)=\left\{\left(p_{0}, p_{1}\right),\left(p_{1}, p_{2}\right),\left(p_{3}, p_{2}\right),\left(p_{0}, p_{3}\right)\right\}$ are the open bounding edges of $G(p)$. The grid partition over $\Omega$ is the set $G(\Omega)=\{G(p) \mid p \in \Omega\}$. Compared to their crisp counterparts, fuzzy vertices, edges, unit areas, grid units, and grid partitions are additionally annotated with finite lists of labels for the sets of membership values according to the definitions given in this paper. Hence, $C_{f}(p)=\left\{\left((p, q),\left\langle\alpha_{j}, \ldots, \alpha_{k}\right\rangle\right)\right\}, \quad V_{f}(p)=\left\{\left(p_{0},\left\langle\alpha_{j}^{0}, \ldots, \alpha_{k}^{0}\right\rangle\right), \ldots,\left(p_{3},\left\langle\alpha_{j}^{3}, \ldots, \alpha_{k}^{3}\right\rangle\right)\right\}, \quad$ and $\quad E_{f}(p)=$ $\left\{\left(\left(p_{0}, p_{1}\right),\left\langle\alpha_{j}^{01}, \ldots, \alpha_{k}^{01}\right\rangle\right), \ldots,\left(\left(p_{0}, p_{3}\right),\left\langle\alpha_{j}^{03}, \ldots, \alpha_{k}^{03}\right\rangle\right)\right\}$ with $1<j \leqslant k \leqslant m$.

A comparison of the implementation of realms (Section 2.3) and grid partitions reveals some differences. Both structures have in common that they are based on the finite point set $\Omega$. But whereas the edge set of a realm is a subset of $\Omega \times \Omega$ with arbitrary but non-intersecting edges, the edge set of a grid partition is much more constrained and represents the complete set of horizontal and vertical unit segments definable over $\Omega$. Hence, a realm usually describes an irregular structure whereas grid partitions are characterized by a regular (unit) structure.

Since segments coming from an application may intersect, they have first both to be redrawn, that is, intersected with each other, before they are accepted in the realm. Such application-based (and, of course, expensive) intersections cannot arise in grid partitions. Since an edge together with its two end points corresponds to a vertical or horizontal unit segment, any two (closed) edges can at most share a common vertex or be identical; they cannot properly intersect. Due to this fact and due to its regularity and completeness, a grid partition does not have to be stored explicitly and persistently like realms, since its complete structure is known in advance. Hence, a grid partition can be regarded as a virtual regular structure which we have to keep in mind when we construct objects but which we do not have to make persistent.

\section{Conclusions}

This paper lays the conceptual and formal foundation for the treatment and implementation of spatial objects blurred by the feature of fuzziness and defined over a discrete geometric domain. The result is a finite resolution fuzzy spatial algebra including fuzzy points, fuzzy lines, and fuzzy regions and also some pertaining operations and predicates. The belief that simply finite point sets are sufficient to appropriately model discrete spatial objects has turned out to be a fallacy. Grid partitions are an appropriate geometric domain for discrete crisp and fuzzy spatial objects since they distinguish different components of space, each component having different dimension. The embedding of grid partitions into the Euclidean space as a topological space enables us to reason about topological relationships between discrete fuzzy regions with the aid of the 9-intersection model. For image data processing the consequence is that the concept of a pixel has to be replaced by the concept of a grid unit. Using this approach this paper contributes to a reduction of the conceptual gap between vector and raster data.

In the future the integration of fuzzy topological predicates into fuzzy spatial query languages will be a subject of further research. The membership degree yielded by a fuzzy topological predicate is a computationally determined quantification. It is thus not very comfortable and userfriendly to use such a value in spatial queries. A solution could be to embed corresponding 
qualitative linguistic descriptions of topological relationships as appropriate interpretations of the membership values into spatial query languages. For instance, depending on the membership value yielded by the predicate inside $_{f}$, we could distinguish between a little bit inside, somewhat inside, quite inside, nearly completely inside, and completely inside. These linguistic terms could then be used in spatial queries.

\section{References}

[1] D. Altman, Fuzzy set theoretic approaches for handling imprecision in spatial analysis, International Journal of Geographical Information Systems 8 (3) (1994) 271-289.

[2] J.E. Bresenham, An Algorithm for Computer Control of a Digital Plotter 4 (1) (1965) 25-30.

[3] P.A. Burrough, Natural objects with indeterminate boundaries, in: P.A. Burrough, A.U. Frank (Eds.), Geographic Objects with Indeterminate Boundaries, GISDATA Series, vol. 2, Taylor \& Francis, London, 1996, pp. 3-28.

[4] P.A. Burrough, A.U. Frank (Eds.), Geographic Objects with Indeterminate Boundaries, GISDATA Series, vol. 2 , Taylor \& Francis, London, 1996.

[5] E. Clementini, P. Di Felice, An algebraic model for spatial objects with indeterminate boundaries, in: P.A. Burrough, A.U. Frank (Eds.), Geographic Objects with Indeterminate Boundaries, GISDATA Series, vol. 2, Taylor \& Francis, London, 1996, pp. 153-169.

[6] F.H. Croom, Basic Concepts of Algebraic Topology, Springer-Verlag, Berlin, 1978.

[7] D. Dubois, M.-C. Jaulent, A general approach to parameter evaluation in fuzzy digital pictures, Pattern Recognition Letters (1987) 251-259.

[8] S. Dutta, Qualitative Spatial Reasoning: A Semi-Quantitative Approach Using Fuzzy Logic, 1st International Symposium on the Design and Implementation of Large Spatial Databases, LNCS 409, Springer-Verlag, 1989, pp. 345-364.

[9] M.J. Egenhofer, A Formal Definition of Binary Topological Relationships, 3rd International Conference on Foundations of Data Organization and Algorithms, LNCS 367, Springer-Verlag, 1989, pp. 457-472.

[10] M.J. Egenhofer, A. Frank, J.P. Jackson, A Topological Data Model for Spatial Databases, 1st International Symposium on the Design and Implementation of Large Spatial Databases, LNCS 409, Springer-Verlag, 1989, pp. 271-286.

[11] M.J. Egenhofer, J. Sharma, Topological Relations between Regions in $\mathbb{R}^{2}$ and $\mathbb{Z}^{2}$, 3rd International Symposium on Advances in Spatial Databases, LNCS 692, Springer-Verlag, 1993, pp. 316-336.

[12] M. Erwig, M. Schneider, Partition and Conquer, 3rd International Conference on Spatial Information Theory, LNCS 1329, Springer-Verlag, 1997, pp. 389-408.

[13] M. Erwig, M. Schneider, Vague Regions, 5th International Symposium on Advances in Spatial Databases, LNCS 1262, Springer-Verlag, 1997, pp. 298-320.

[14] A. Frank, W. Kuhn, Cell Graphs: A Provable Correct Method for the Storage of Geometry, 3rd International Symposium on Spatial Data Handling, 1986, pp. 411-436.

[15] S. Gaal, Point Set Topology, Academic Press, London, 1964.

[16] D. Greene, F. Yao, Finite-Resolution Computational Geometry, 27th IEEE Symposium on Foundations of Computer Science, 1986, pp. 143-152.

[17] R.H. Güting, M. Schneider, Realms: A Foundation for Spatial Data Types in Database Systems, 3rd International Symposium on Advances in Spatial Databases, LNCS 692, Springer-Verlag, 1993, pp. 14-35.

[18] R.H. Güting, M. Schneider, Realm-based spatial data types: the ROSE algebra, VLDB Journal 4 (1995) 100-143.

[19] T.Y. Kong, A. Rosenfeld, Digital topology: introduction and survey, Computer Vision, Graphics, and Image Processing 48 (1989) 357-393.

[20] A. Rosenfeld, Fuzzy digital topology, Information and Control 40 (1979) 76-87.

[21] M. Schneider, Modelling spatial objects with undetermined boundaries using the Realm/ROSE approach, in: P.A. Burrough, A.U. Frank (Eds.), Geographic Objects with Indeterminate Boundaries GISDATA Series, vol. 2, Taylor \& Francis, London, 1996, pp. 141-152. 
[22] M. Schneider, Spatial Data Types for Database Systems-Finite Resolution Geometry for Geographic Information Systems, vol. LNCS 1288, Springer-Verlag, Berlin, 1997.

[23] M. Schneider, Uncertainty Management for Spatial Data in Databases: Fuzzy Spatial Data Types, 6th International Symposium on Advances in Spatial Databases, LNCS 1651, Springer-Verlag, Berlin, 1999, pp. 330351.

[24] M. Schneider, Finite Resolution Crisp and Fuzzy Spatial Objects, International Symposium on Spatial Data Handling, 2000, pp. 5a.3-17.

[25] R. Shibasaki, A Framework for Handling Geometric Data with Positional Uncertainty in a GIS Environment, GIS: Technology and Applications, World Scientific, Singapore, 1993, pp. 21-35.

[26] M. Worboys, Imprecision in finite resolution spatial data, GeoInformatica 2 (3) (1998) 257-279.

[27] L.A. Zadeh, Fuzzy sets, Information and Control 8 (1965) 338-353.

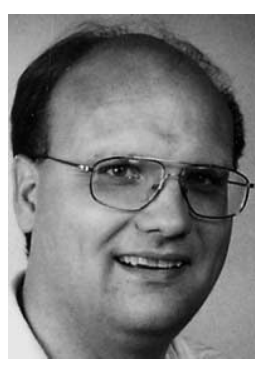

Markus Schneider received his Diploma degree in computer science from the University of Dortmund, Germany, in 1990, and his Ph.D. degree (Dr. rer. nat.) in computer science from the University of Hagen, Germany, in 1995. After that, until 2001, he was research assistant (lecturer) at the University of Hagen. Since 2002 he has been an Assistant Professor at the University of Florida, Gainesville, FL, USA. His research interests are spatial, spatio-temporal, and fuzzy databases. In particular, he focuses on the design and implementation of spatial data types (geo-relational algebra, ROSE algebra, realms), spatio-temporal data types (moving objects), fuzzy spatial objects, spatial and spatio-temporal partitions and networks, and deals with the design and implementation of data structures and geometric algorithms for these topics. 